\title{
AUTOMORPHISMS OF SIEGEL DOMAINS
}

\author{
BY \\ O. S. ROTHAUS( $\left.{ }^{1}\right)$
}

\begin{abstract}
This paper studies nonaffine biholomorphisms from one tube domain to a second. A sequel will carry out the same study for arbitrary Siegel domains. With the help of the Bergman kernel function, we can give an explicit form for such biholomorphisms; and with the use of structure theory for Jordan algebras, we can give an algebraic and geometric description of the nature of such tube domains.
\end{abstract}

I. Introduction. As is well known [10], every bounded complex homogeneous domain in the space of $n$ complex variables is holomorphically equivalent to a Siegel domain of first or second kind. This being the case, it is of some interest to investigate the holomorphic bijections from one such domain to another, even without the assumption of homogeneity for either. This investigation has been, from various points of view, the object of several recent papers [3], [4], [9]. In this paper, we use a simple procedure, already seen in a particular case in [7], for studying this question for domains of the first kind; i.e., tube domains. This study is a necessary preliminary to the investigation of the general case, which we hope to present in a projected sequel to this paper. Our method readily yields most of the known results, as well as several new ones. For example, we can show that if a tube domain, not assumed homogeneous, has a single holomorphic involution with an isolated fixed point, it is already a Hermitian symmetric space.

Our method depends principally on the explicit construction of the Bergman kernel function for Siegel domains, without the assumption of homogeneity. This is already known for tube domains, but in anticipation of the sequel to this paper, we construct it anew for general Siegel domains. An easy by-product of the analysis is the known conclusion [4] that holomorphically equivalent Siegel domains are already affinely equivalent. With a little more care, it is even possible to completely specify all the holomorphic automorphisms of a Siegel domain, though, as we have said before, we concentrate in this paper only on tube domains. Analogously, the infinitesimal generators of the group of holomorphic automorphisms may also be completely specified.

Briefly, the outline of this paper is as follows. §I sets some notation and establishes a few simple facts about the distinguished boundary of Siegel domains. In $\S I$, we construct the kernel function and indicate how we propose to make use of

Received by the editors August 31, 1970 and, in revised form, April 6, 1971.

AMS 1970 subject classifications. Primary 32A07; Secondary 17B60.

Key words and phrases. Convex cone, tube domain, Bergman kernel, Jordan algebra.

${ }^{(1)}$ This research was partially supported by NSF GP-8129.

Copyright (C) 1972, American Mathematical Society 
it. In §III, we assume we have a nonaffine biholomorphism from one tube domain over a cone $C$ to a second tube domain. From this assumption alone, and an explicit formula for the biholomorphism gained through use of the kernel function, we are able to conclude that the ambient real linear space of $C$ has the structure of a Jordan algebra such that Jordan multiplication is an infinitesimal automorphism of $C$. In §IV we take the last conclusion as hypothesis, characterize all such cones, and show that the tube over such cones has nonaffine automorphisms. In $\S \mathrm{V}$, we study the Lie algebra of the group of automorphisms of a tube domain, and essentially integrate all the infinitesimal motions.

Since we depart slightly from the usual conventions, and in order to fix notation, it seems appropriate to begin by defining Siegel domains. Let $\boldsymbol{C}^{m}=\boldsymbol{R}^{m} \oplus i \boldsymbol{R}^{m}$, $\boldsymbol{C}^{n}=\boldsymbol{R}^{n} \oplus i \boldsymbol{R}^{n}$ be the usual $m$ and $n$ dimensional complex spaces respectively. Complex conjugation and real part shall be taken in both cases with respect to the underlying real subspace. Let $C$ be a regular open convex cone in $\boldsymbol{R}^{m}$. Regular means that $\bar{C}$ does not contain an entire straight line. Let $H$ be a $C^{m}$-Hermitian form on $\boldsymbol{C}^{n}$ with respect to $C$, i.e. $H$ is a function from $\boldsymbol{C}^{n} \times \boldsymbol{C}^{n}$ to $\boldsymbol{C}^{m}$ satisfying

(i) $H\left(w_{1}, w_{2}\right)=\bar{H}\left(\bar{w}_{2}, \bar{w}_{1}\right)$ for $w_{1}, w_{2} \in \boldsymbol{C}^{n}$.

(ii) $H\left(\alpha w_{1}+\beta w_{2}, w_{3}\right)=\alpha H\left(w_{1}, w_{3}\right)+\beta H\left(w_{2}, w_{3}\right)$ for $\alpha, \beta \in C, w_{1}, w_{2}, w_{3} \in C^{n}$.

(iii) $H(w, \bar{w}) \in \bar{C}$ (closure of $C$ ) and $H(w, \bar{w})=0$ if and only if $w=0$.

Then the set of points $(z, w) \in C^{m} \times C^{n}$ with $\operatorname{Re} z-H(w, \bar{w}) \in C$ is called a Siegel domain of second kind. A Siegel domain of first kind, or a tube domain, is the special case in which $n=0$ and $H \equiv 0$.

The cone $C$ gives rise to a partial order in $\boldsymbol{R}^{m}$, and we write $a>b(a \geqq b)$ if $a-b \in C$ $(a-b \in \bar{C})$. With this notation in hand the Siegel domain $\mathscr{D}$ above may be described by

$$
\mathscr{D}=\left\{(z, w) \in \boldsymbol{C}^{m} \times C^{n} \mid \operatorname{Re} z>H(w, \bar{w})\right\} .
$$

Siegel domains have extensive groups of holomorphic automorphisms. The following three types of maps are always automorphisms:

(i) $z \rightarrow \alpha \bar{\alpha} z, w \rightarrow \alpha w ; \alpha$ an arbitrary nonzero complex number.

(ii) $z \rightarrow z+2 H(w, \bar{b})+H(b, \bar{b}), w \rightarrow w+b$; $b$ being any point in $C^{n}$.

(iii) $z \rightarrow z+i b, w \rightarrow w ; b$ being any point in $\boldsymbol{R}^{m}$.

The distinguished boundary of $\mathscr{D}$ is the set

$$
\mathscr{B}=\left\{(z, w) \in C^{m} \times C^{n} \mid \operatorname{Re} z=H(w, \bar{w})\right\} .
$$

Note that the automorphisms just described act transitively on the distinguished boundary. We need some elementary properties of the distinguished boundary which do not seem to be readily available in the literature.

A sequence $x_{v}$ of points of $\mathscr{D}$ is said to approach the distinguished boundary if either $\lim x_{v}$ exists and is a point of $\mathscr{B}$, or the sequence is unbounded. For a holomorphic function $f$ defined in $\mathscr{D}$, the sequence $x_{v}$ of points in $\mathscr{D}$ is said to be a maximizing sequence if $\lim \left|f\left(x_{v}\right)\right|=\sup _{x \in \mathscr{D}}|f(x)|$. The supremum may be infinite, of course. 
Lemma 1. Let $f$ be a holomorphic function on $\mathscr{D}$. Then there is a maximizing sequence for $f$ which approaches the distinguished boundary.

For the proof, let $u_{v}=\left(z_{v}, w_{v}\right)$ be any sequence such that $\lim \left|f\left(u_{v}\right)\right|=$ $\sup _{u \in \mathscr{D}}|f(u)|$. Let $z_{v}=x_{v}+i y_{v}, x_{v}$ and $y_{v}$ real. Consider the function of a single complex variable $\lambda$,

$$
F_{v}(\lambda)=f\left(H\left(w_{v}, \bar{w}_{v}\right)+\lambda\left(x_{v}-H\left(w_{v}, \bar{w}_{v}\right)\right)+i y_{v}, w_{v}\right)
$$

defined for $\operatorname{Re} \lambda>0$. Note that $F_{v}(1)=f\left(z_{v}, w_{v}\right)$. If the sequence $u_{v}$ is unbounded, we are done. Otherwise, we may assume, going to a subsequence if necessary, that $\lim u_{v}$ exists. If then for infinitely many values of $\nu, F_{v}(\lambda)$ achieves its maximum modulus at " $\infty$ ", then $\lambda_{v}$ may be selected so that $H\left(w_{v}, \bar{w}_{v}\right)+\lambda_{v}\left(x_{v}-H\left(w_{v}, \bar{w}_{v}\right)\right)$ has an unbounded subsequence, and so that $\left|F_{v}\left(\lambda_{v}\right)\right| \geqq\left|F_{v}(1)\right|$. So we are done in this case also. Otherwise, there is a bounded subsequence $\lambda_{v}$ with $\operatorname{Re} \lambda_{v} \rightarrow 0$ and $\left|F_{v}\left(\lambda_{v}\right)\right| \geqq\left|F_{v}(1)\right|$. Going to a convergent subsequence of such a sequence of $\lambda_{v}$ 's, we have that $\left(H\left(w_{v}, \bar{w}_{v}\right)+\lambda_{v}\left(x_{v}-H\left(w_{v}, \bar{w}_{v}\right)\right)+i y_{v}, w_{v}\right)$ is a maximizing sequence converging to a point of the distinguished boundary, which completes the proof.

Let $\mathscr{D}$ and $\mathscr{D}^{\prime}$ be Siegel domains, and $f$ a biholomorphism from $\mathscr{D}$ to $\mathscr{D}^{\prime}$. Let $b$ be a distinguished boundary point of $\mathscr{D}$. $f$ is said to be defined at $b$ if $\lim _{x \rightarrow b} f(x)$ exists for approaches to $b$ through points of $\mathscr{D}$.

LEMMA 2. If $f$ is defined at $b$, then $\lim _{x \rightarrow b} f(x) \in \mathscr{B}^{\prime}$, the distinguished boundary of $\mathscr{D}^{\prime}$.

For the proof, we introduce the usual scalar product $\langle\cdot, \cdot\rangle$ in $\boldsymbol{R}^{m}$. We extend the scalar product by linearity to a bilinear form on $C^{m}$. Let $C^{*}$ be the dual cone to $C$ with respect to above scalar product; i.e.,

$$
C^{*}=\text { Interior }\left\{x \in R^{m} \mid\langle x, c\rangle \geqq 0 \forall c \in C\right\} \text {. }
$$

$C^{*}$ is also an open regular (nonempty) convex cone. Now we consider the function

$$
M(z)=\int_{C^{*}} \exp \langle-z, x\rangle d x .
$$

$M(z)$ is a well-defined [6], [8] holomorphic function for $z \in T_{C}$, the tube over the cone $C$. If $p$ is any point in $C$, it is easy to see that the function $M(z+p)$ is holomorphic in a neighborhood of the closure of $T_{C}$, and achieves its maximum modulus in the closure of $T_{C}$ uniquely at the point $z=0$.

We define a holomorphic function $F(z, w)$ in $\mathscr{D}$ by setting $F(z, w)=M(z+p)$. $F(z, w)$ is defined and holomorphic in a neighborhood of the closure of $\mathscr{D}$ and achieves its maximum modulus in $\overline{\mathscr{D}}$ uniquely at the point $(z, w)=(0,0)$.

Returning now to the proof of the lemma, observe that since the automorphisms of $\mathscr{D}$ are transitive on the distinguished boundary, it suffices to take only the case $b=0$. Consider the function $h=F \circ f^{-1}$. $h$ is holomorphic in $\mathscr{D}^{\prime}$. Let $y_{v}$ be any maximizing sequence for $h$. Then $x_{v}=f^{-1}\left(y_{v}\right)$ is a maximizing sequence for $F$. 
From the properties of $F$ cited above, it follows that $\lim x_{v}=0$. Since $f$ is defined at 0 , and $y_{v}=f\left(x_{v}\right)$, it follows that $\lim y_{v}$ exists. Since by the last lemma, it is possible to have selected the maximizing sequence $y_{v}$ to approach the distinguished boundary of $\mathscr{D}^{\prime}$, it follows that $\lim y_{v} \in \mathscr{B}^{\prime}$.

Finally we have

LEMMA 3. If $f$ is holomorphic in $\mathscr{D}$, is defined and continuous in a neighborhood $N$ of a point $b \in \mathscr{B}$, and vanishes on $N \cap B$, then $f$ is identically 0 .

For the proof, it suffices as before to take only the case $b=0$. For fixed $(z, w) \in \mathscr{D}$, $z=x+i y$, we consider the function of the single complex variable $\lambda$ given by

$$
F(\lambda)=f(H(w, \bar{w})+\lambda(x-H(w, \bar{w}))+i y, w)
$$

and note that $F(1)=f(z, w) . F$ is defined and holomorphic for $\operatorname{Re} \lambda>0$. What is more, if $w$ is sufficiently close to the origin, then $F(\lambda)$ is continuous in a neighborhood of $\lambda=0$, and vanishes on the imaginary axis in a neighborhood of $\lambda=0$. Hence for such $w, F(\lambda) \equiv 0$, and this is enough to prove $f \equiv 0$.

II. The kernel function. Since $C$ is a regular cone, we may by an appropriate coordinate change arrange that $C$ is contained in the first $m$-gant in $\boldsymbol{R}^{m}$. We will suppose this has been done. Consider now the function

$$
K_{\varepsilon}(z)=\left\{\left(1+\varepsilon z_{1}\right)\left(1+\varepsilon z_{2}\right) \cdots\left(1+\varepsilon z_{m}\right)\right\}^{-2}, \quad \text { where } \varepsilon>0 \text { and } z \in T_{C} .
$$

Our supposition above on $C$ guarantees that $K_{\varepsilon}(z)$ is integrable and square integrable in $T_{C}$. Moreover $\left|K_{\varepsilon}(z)\right|<1$.

Now let $F(z, w)$ be a square integrable holomorphic function in $\mathscr{D}$. Consider the function $F_{\varepsilon}(z, w)=K_{\varepsilon}(z) F(z, w)$. It is clear that $\lim _{\varepsilon \rightarrow 0} F_{\varepsilon}(z, w)=F(z, w)$ in the $L_{2}$ mean for $\mathscr{D}$. And the function $F_{\varepsilon}(z, w)$ has some exceedingly useful properties.

For any compactum $A$ in $C$ put

$$
\mathscr{D}_{A}=\{(z, w) \in \mathscr{D} \mid \operatorname{Re} z-H(w, \bar{w}) \in A\} .
$$

The distance of the points of the set $\mathscr{D}_{A}$ from the boundary of $\mathscr{D}$ is uniformly bounded away from zero. Hence it follows from standard arguments that $|F(z, w)|$ is bounded for $(z, w) \in \mathscr{D}_{A}$. Put $z=x+i y$. Thus for $(z, w) \in \mathscr{D}$ we may form the Fourier transform

$$
g_{\varepsilon}(t, x, w)=\int_{R^{m}} F_{\varepsilon}(z, w) \exp \langle i y, t\rangle d y .
$$

On the other hand, another standard argument, which we have used before [6], [8], shows that in fact

$$
g_{\varepsilon}(t, x, w)=G_{\varepsilon}(t, w) \exp \langle-x, t\rangle
$$

where

$$
G_{\varepsilon}(t, w)=\int_{R^{m}} F_{\varepsilon}(z, w) \exp \langle z, t\rangle d y
$$


Since the integral defining $G_{\varepsilon}$ is uniform in $w$, for $w$ in any compactum in $C^{m}$, it follows as well that $G_{\varepsilon}(t, w)$ is an entire function of $w$ for any fixed value of $t$. Now by Plancherel's theorem

$$
\int_{R^{m}}\left|F_{\varepsilon}(z, w)\right|^{2} d y=(2 \pi)^{m} \int_{R^{m}} \exp \langle-2 x, t\rangle \cdot\left|G_{\varepsilon}(t, w)\right|^{2} d t .
$$

Since $F_{\varepsilon} \in L_{2}(\mathscr{D})$, we must have that $\int\left|F_{\varepsilon}(z, w)\right|^{2} d y d x$, where the integration is over all $y \in \boldsymbol{R}^{m}$, and all $x>H(w, \bar{w})$, is finite for almost all $w$. But if $t \notin C^{*}$, then we have that $\int_{x>H(w, \bar{w})} \exp \langle-2 x, t\rangle d x$ is infinite for any $w$. Hence we may conclude that $G_{\varepsilon}(t, w)$ is zero for almost all $t \notin C^{*}$, and since $G_{\varepsilon}$ is a continuous function of $(t, w), G_{\varepsilon}(t, w)$ is zero for all $t \notin C^{*}$.

By Fourier inversion, we may write now

$$
F_{\varepsilon}(z, w) \simeq \frac{1}{(2 \pi)^{m}} \int_{C^{*}} G_{\varepsilon}(t, w) \exp \langle-z, t\rangle d t
$$

which holds as an ordinary equality, by virtue of the following argument. We know that $x>H(w, \bar{w})$, so for some $\eta, 0<\eta<1, \eta x>H(w, \bar{w})$. Then

$$
\begin{aligned}
\int_{C^{*}} \mid G_{\varepsilon}(t, w) \exp & \langle-z, t\rangle \mid d t \\
& =\int_{C^{*}}\left|G_{\varepsilon}(t, w)\right| \exp (-\eta\langle x, t\rangle) \exp ((\eta-1)\langle x, t\rangle) d t \\
& \leqq\left\{\int_{C^{*}}\left|G_{\varepsilon}(t, w)\right|^{2} \exp (-2 \eta\langle x, t\rangle) d t \cdot \int_{C^{*}} \exp 2(\eta-1)\langle x, t\rangle d t\right\}^{1 / 2} \\
& =\left\{\frac{1}{(2 \pi)^{m}} \int_{R^{m}}\left|F_{\varepsilon}(\eta x+i y, w)\right|^{2} d y \cdot \int_{C^{*}} \exp 2(\eta-1)\langle x, t\rangle d t\right\}^{1 / 2}<\infty
\end{aligned}
$$

which shows that the integral exists in the ordinary sense. The same argument shows, moreover, that the integral is uniform for $(z, w)$ in a compactum in $\mathscr{D}$, which establishes the desired equality.

Now let $M(t)=\int_{C} \exp \langle-t, x\rangle d x . M(t)$ is well defined for $t \in C^{*}$.

For any $t \in \boldsymbol{R}^{m}$, we define a matrix $\Theta(t)$ by $\left\langle H\left(w_{1}, w_{2}\right), t\right\rangle=\left\langle w_{2}, \Theta(t) w_{1}\right\rangle$. Because of the properties of $H$ cited in $\S \mathrm{I}, \Theta(t)$ is Hermitian symmetric, and is Hermitian positive definite for $t \in C^{*}$. Then let

$$
L(z)=\int_{C^{*}} \exp \langle-z, t\rangle \cdot \frac{1}{M(t)} \operatorname{det} \Theta(t) d t .
$$

The integral is a well-defined holomorphic function of $z$ for $z \in T_{C}$, by virtue of estimates available in [8] for $M(t)$ and the obvious fact that det $\Theta(t)$ is dominated by a finite power of $\langle t, t\rangle$. We claim

THEOREM 1. $\mathscr{B}((Z, W) ;(z, w))=\left(1 / \pi^{m+n}\right) L(Z+\bar{z}-2 H(W, \bar{w}))$ is the Bergman kernel function for $\mathscr{D}$. 
We have only to show that $\mathscr{B}$ is defined, is for fixed $(Z, W)$ in $\mathscr{D}$ a square integrable conjugate holomorphic function of $(z, w)$ in $\mathscr{D}$, and that for every holomorphic square integrable $F$ in $\mathscr{D}$, we have

$$
\int_{\mathscr{D}} \mathscr{B}((Z, W) ;(z, w)) F(z, w) d z d w=F(Z, W)
$$

That $\mathscr{B}$ is defined follows from the observation that $Z+\bar{z}-2 H(W, \bar{w}) \in T_{C}$. That $\mathscr{B}$ is square integrable follows easily with use of Plancherel's theorem, and is left to the reader. For the final statement, it suffices to show that for all $\varepsilon>0$, we have

$$
\int_{\mathscr{D}} \mathscr{B} \cdot F_{\varepsilon}(z, w) d z d w=F_{\varepsilon}(Z, W)
$$

Now we may use the formula (I) developed above for $F_{\varepsilon}$, and apply first Plancherel's theorem.

$$
\begin{aligned}
& \int \mathscr{B} F_{\varepsilon}(z, w) d y \\
& \quad=\frac{1}{\pi^{m+n}} \int_{C^{*}} \exp (-\langle Z, t\rangle-2\langle x, t\rangle+2\langle H(W, \bar{w}), t\rangle) G_{\varepsilon}(t, w) \frac{1}{M(t)} \operatorname{det} \Theta(t) d t .
\end{aligned}
$$

Now holding $w$ fixed, integrate over those $x$ for which $x>H(w, \bar{w})$. We obtain

$$
\begin{aligned}
& \int_{x>H(w, \bar{w})} \mathscr{B} \cdot F_{\varepsilon}(z, w) d y d x \\
&= \frac{1}{2^{m} \pi^{m+n}} \int_{C^{*}} \exp (-\langle Z, t\rangle-2\langle H(w, \bar{w}), t\rangle+2\langle H(W, \bar{w}), t\rangle) \\
& \cdot G_{\varepsilon}(t, w) \operatorname{det} \Theta(t) d t .
\end{aligned}
$$

Now we want to evaluate

$$
\int_{C^{n}} \exp (-2\langle H(w, \bar{w}), t\rangle+2\langle H(W, \bar{w}), t\rangle) G_{\varepsilon}(t, w) d w
$$

It will suffice to evaluate when $W=0$, the general case being taken care of by replacing $w$ by $w+W$. For the special case, we replace in the integral $w$ by $\Theta^{1 / 2}(t) w$, where $\Theta^{1 / 2}(t)$ is the Hermitian definite square root of $\Theta(t)$. The integral in question then becomes

$$
1 / \operatorname{det} \Theta(t) \int_{C^{n}} \exp \langle-2 w, \bar{w}\rangle G_{\varepsilon}\left(t, \Theta^{-1 / 2}(t) w\right) d w .
$$

If we now write $w=u+i v$, replace each pair of components of $(u, v)$ by polar coordinates, then expand $G_{\varepsilon}\left(t, \Theta^{-1 / 2}(t) w\right)$ in a power series in $w$, we find immediately that only the constant term of the series contributes to the integral. To be completely precise, we find for the general case that (II) above is equal to 
$\left(\pi^{n} / \operatorname{det} \Theta(t)\right) \cdot G_{\varepsilon}(t, W)$. Hence finally,

$$
\int_{\mathscr{D}} \mathscr{B} F_{\varepsilon}(z, w) d z d w=\frac{1}{(2 \pi)^{m}} \int_{C^{\bullet}} \exp \langle-z, t\rangle G_{\varepsilon}(t, W) d t=F_{\varepsilon}(Z, W)
$$

which completes the proof.

We must note that the formula is valid as it stands, without the provision made at the outset that $C$ lies in the first $m$-gant. This assumption was used only to construct the functions $K_{\varepsilon}(z)$, which could obviously have been constructed in a slightly more complicated fashion without the use of this assumption. The remaining manipulations are generally valid.

Also, we want to observe that for the case of tube domains, the formula for the kernel function agrees with the known result, provided we take det $\Theta(t) \equiv 1$.

It is probably also worth noting that the general nature of the kernel function for $\mathscr{D}$, at least to an extent sufficient for our purposes, can be discovered from functorial properties alone.

For let $\mathscr{B}((Z, W) ;(z, w))$ be the kernel function, $\mathscr{B}$ holomorphic in the variables $(Z, W)$ and conjugate holomorphic in the variables $(z, w)$. If $T: \mathscr{D} \leftrightarrow \mathscr{D}$ is a biholomorphism, then we must have

$$
\mathscr{B}(T(Z, W) ; T(z, w))\left\|\frac{\partial T(Z, W)}{\partial(Z, W)}\right\|\left\|\overline{\frac{\partial T(z, w)}{\partial(z, w)}}\right\|=\mathscr{B}((Z, W) ;(z, w))
$$

where $\|\partial T(Z, W) / \partial(Z, W)\|$ is the determinant of the Jacobian of $T$.

If we now let $T$ be an automorphism of type (iii) described in $\S$ I, then we obtain

$$
\mathscr{B}((Z+i b, W) ;(z+i b, w))=\mathscr{B}((Z, W) ;(z, w)) .
$$

From this it follows easily by analytic continuation that in fact

$$
\mathscr{B}((Z, W) ;(z, w))=\mathscr{B}^{\prime}(Z+\bar{z} ; W, w)
$$

for some function $\mathscr{B}^{\prime}$, which is holomorphic in the first two variables and conjugate holomorphic in the third.

Now apply an automorphism of type (ii). Then we obtain

$$
\begin{gathered}
\left.\mathscr{B}^{\prime}(Z+2 H(W, \bar{b})+H(b, \bar{b})+\bar{z}+2 \overline{H(w, \bar{b}})+H(b, \bar{b}), W+b, w+b\right) \\
=\mathscr{B}^{\prime}(Z+\bar{z}, W, w)
\end{gathered}
$$

for any $b$ in $C^{n}$. Put $b=-w$ to yield

$$
\mathscr{B}^{\prime}(Z+\bar{z}, W, w)=\mathscr{B}^{\prime}(Z+\bar{z},-2 H(W, \bar{w}), W-w, 0) .
$$

Put $W=0$ in the last. We obtain $\mathscr{B}^{\prime}(Z+\bar{z}, 0, w)=\mathscr{B}^{\prime}(Z+\bar{z},-w, 0)$. Since the function on the right is holomorphic in $w$, and the function on the left is conjugate holomorphic in $w$, we conclude that $\mathscr{B}^{\prime}(z, w, 0)$ is a constant function of the second 
variable. Thus,

$$
\begin{aligned}
\mathscr{B}^{\prime}(Z+\bar{z}, W, w) & =\mathscr{B}^{\prime}(Z+\bar{z}-2 H(W, \bar{w}), W-w, 0) \\
& =\mathscr{B}^{\prime}(Z+\bar{z}-2 H(W, \bar{w}), 0,0)=\mathscr{B}^{\prime \prime}(Z+\bar{z}-2 H(W, \bar{w}))
\end{aligned}
$$

for a holomorphic function $\mathscr{B}^{\prime \prime}$ which must be defined in $T_{C}$.

Finally, using automorphisms of type (i), it is clear that $\mathscr{B}^{\prime \prime}$ is homogeneous of degree $-(2 m+n)$.

Our use of the kernel function will be as follows. Let $\mathscr{D}_{1}$ and $\mathscr{D}_{2}$ be domains in $C^{k}, \mathscr{B}_{1}\left(z_{1}, z_{2}\right)$ and $\mathscr{B}_{2}\left(w_{1}, w_{2}\right)$ their respective Bergman kernel functions. $\mathscr{B}_{1}$ is holomorphic in $z_{1}$ and conjugate holomorphic in $z_{2}$, and analogously for $\mathscr{B}_{2}$. We assume the existence of a biholomorphism $w=f(z)$ from $\mathscr{D}_{1}$ to $\mathscr{D}_{2}$. From general properties of kernel functions, we obtain then a useful statement (Theorem 2) about the map $w=f(z)$ which, specialized to the case $\mathscr{D}_{1}$ and $\mathscr{D}_{2}$ Siegel domains and $w$ nonaffine, yields precise information both about the map and the geometric structure of $\mathscr{D}_{1}$ or $\mathscr{D}_{2}$.

First we want to introduce a little notation. If $w=g(z)$ is a differentiable function from $\boldsymbol{C}^{k}$ to $\boldsymbol{C}^{l}$, then by $\partial w / \partial z$ we shall denote the matrix whose $i$ th row and $j$ th column is $\partial w^{i} / \partial z^{j}$. If $A$ is a matrix, ${ }^{t} A$ denotes its transpose. If $A$ is square, $|A|$ will denote the determinant of $A$. If $x=g(z, w)$ is a differentiable function from $\boldsymbol{C}^{k} \times C^{l}$ to $C$, then by $\partial^{2} x / \partial z \partial w$ we denote the matrix whose $i$ th row and $j$ th column is $\partial^{2} x / \partial z^{i} \partial w^{j}$

From well-known properties of the kernel function we have

$$
\mathscr{B}_{2}\left(w_{1}, w_{2}\right)\left|\frac{\partial w_{1}}{\partial z_{1}}\right|\left|\overline{\frac{\partial w_{2}}{\partial z_{2}}}\right|=\mathscr{B}_{1}\left(z_{1}, z_{2}\right)
$$

Assume for the moment that $\left(z_{1}, z_{2}\right)$ is such that $\mathscr{B}_{1}\left(z_{1}, z_{2}\right) \neq 0$, which guarantees that $\mathscr{B}_{2}\left(w_{1}, w_{2}\right) \neq 0$. Put $b_{1}\left(z_{1}, z_{2}\right)=\left(1 / \mathscr{B}_{1}\right) \cdot \partial \mathscr{B}_{1} / \partial z_{1}$. Analogously, put $b_{2}\left(w_{1}, w_{2}\right)$ $=\left(1 / \mathscr{B}_{2}\right) \cdot \partial \mathscr{B}_{2} / \partial w_{1}$. Then differentiating the relation (III) above with respect to $z_{1}$, we may write

$$
{ }^{t}\left(\frac{\partial w_{1}}{\partial z_{1}}\right) b_{2}\left(w_{1}, w_{2}\right)+\left|\frac{\partial w_{1}}{\partial z_{1}}\right|^{-1} \frac{\partial}{\partial z_{1}}\left|\frac{\partial w_{1}}{\partial z_{1}}\right|=b_{1}\left(z_{1}, z_{2}\right)
$$

Let $B_{1}\left(z_{1}, z_{2}\right)=\partial b_{1}\left(z_{1}, z_{2}\right) / \partial \bar{z}_{2}$, and analogously define $B_{2}\left(w_{1}, w_{2}\right) . B_{1}$ is holomorphic in $z_{1}$ and conjugate holomorphic in $z_{2}$ on the complement of the set $\left(z_{1}, z_{2}\right)$ for which $\mathscr{B}_{1}\left(z_{1}, z_{2}\right)=0$. In general the square matrices $B_{1}$ and $B_{2}$ are nonsingular, since they are defined and nonsingular on the diagonal. The set of $\left(z_{1}, z_{2}\right)$ for which $\mathscr{B}_{1}\left(z_{1}, z_{2}\right) \neq 0$ and $B_{1}\left(z_{1}, z_{2}\right)$ is nonsingular is the complement of a globally defined "analytic" variety in $C^{k} \times C^{k}$, so does not disconnect the space. We call the complement the regular set $R$ in $C^{k} \times C^{k}$. (That the functions entering the statements above are all conjugate holomorphic in some of the variables does not affect any of the usual conclusions.) 
If we differentiate (IV) with respect to $\bar{z}_{2}$ we obtain

$$
{ }^{t}\left(\partial w_{1} / \partial z_{1}\right) B_{2}\left(w_{1}, w_{2}\right)\left(\overline{\partial w_{2} / \partial z_{2}}\right)=B_{1}\left(z_{1}, z_{2}\right)
$$

which is valid for $\left(z_{1}, z_{2}\right) \in R$.

Let $p$ be a fixed point in $\mathscr{D}_{1}$, and put $q=f(p)$. Subtract from (IV) the same relation with $z_{2}$ replaced by $p$, to obtain

$$
{ }^{t}\left(\partial w_{1} / \partial z_{1}\right)\left\{b_{2}\left(w_{1}, w_{2}\right)-b_{2}\left(w_{1}, q\right)\right\}=b_{1}\left(z_{1}, z_{2}\right)-b_{1}\left(z_{1}, p\right)
$$

which is valid for example for each of $z_{1}$ and $z_{2}$ in some neighborhood of $p$.

For $\left(z_{1}, z_{2}\right) \in R$ we may define a function $c_{1}\left(z_{1}, z_{2}\right)$ by

$$
B_{1}\left(z_{1}, z_{2}\right) c_{1}\left(z_{1}, z_{2}\right)=b_{1}\left(z_{1}, z_{2}\right) \text {. }
$$

Analogously for $\left(w_{1}, w_{2}\right)$ in the image of $R$ under map $(f, f)$, we may define $c_{2}\left(w_{1}, w_{2}\right)$ by

$$
B_{2}\left(w_{1}, w_{2}\right) c_{2}\left(w_{1}, w_{2}\right)=b_{2}\left(w_{1}, w_{2}\right)
$$

Now using these definitions in conjunction with (V) and (VI) we obtain

$$
\begin{aligned}
B_{1}\left(z_{1}, z_{2}\right)\left\{\left(\overline{\partial w_{2} / \partial z_{2}}\right)^{-1} c_{2}\left(w_{1}, w_{2}\right)-c_{1}\left(z_{1}, z_{2}\right)\right\} \\
=B_{1}\left(z_{1}, p\right)\left\{\left(\overline{\partial w_{2} / \partial z_{2}}\right)^{-1}(p) c_{2}\left(w_{1}, q\right)-c_{1}\left(z_{1}, p\right)\right\}
\end{aligned}
$$

which holds for each of $z_{1}, z_{2}$ in some neighborhood of $p$. Then using analytic continuation we conclude

THEOREM 2. The function

$$
B_{1}\left(z_{1}, z_{2}\right)\left\{\left(\partial w_{2} / \partial z_{2}\right)^{-1} c_{2}\left(w_{1}, w_{2}\right)-c_{1}\left(z_{1}, z_{2}\right)\right\}
$$

is defined for $\left(z_{1}, z_{2}\right) \in R$ and is independent of $z_{2}$.

In the particular applications we make of the above theorem, the functions $c_{1}$ and $c_{2}$ will extend to be defined everywhere, so that the restriction $\left(z_{1}, z_{2}\right) \in R$ may be somewhat relaxed.

III. Biholomorphisms of tube domains. We begin by applying the considerations of the last section to the case in which both $\mathscr{D}_{1}$ and $\mathscr{D}_{2}$ are tube domains over regular convex cones $C_{1}$ and $C_{2}$ respectively.

We know that $\mathscr{B}_{i}\left(z_{1}, z_{2}\right)=L_{i}\left(z_{1}+\bar{z}_{2}\right)(i=1,2)$ and so we may take $B_{i}\left(z_{1}, z_{2}\right)$ $=B_{i}\left(z_{1}+\bar{z}_{2}\right)$ as function of sum of the variables only.

Since $L_{i}$ is a homogeneous function, it follows immediately that we may take $c_{i}\left(z_{1}, z_{2}\right)=-\left(z_{1}+\bar{z}_{2}\right)$. So we have the following statement:

$$
B_{1}\left(z_{1}+\bar{z}_{2}\right)\left\{\left(\overline{\partial w_{2} / \partial z_{2}}\right)^{-1}\left(w_{1}+\bar{w}_{2}\right)-\left(z_{1}+\bar{z}_{2}\right)\right\}
$$

is a function of $z_{1}$ alone and is defined provided $L_{1}\left(z_{1}+\bar{z}_{2}\right) \neq 0$. 
Now let $z_{2}^{0}$ be an arbitrary point in $C^{m}$. Select $z_{1}^{0} \in T_{C_{1}}$ so that $z_{1}^{0}+\bar{z}_{2}^{0} \in T_{C_{1}}$, so that $L_{1}\left(z_{1}^{0}+\bar{z}_{2}^{0}\right) \neq 0$, and so that $B_{1}\left(z_{1}^{0}+\bar{z}_{2}^{0}\right)$ is nonsingular. It is easy enough to so choose $z_{2}^{0}$. Then $\left[B_{1}\left(z_{1}^{0}+\bar{z}_{2}\right)\right]^{-1}$ is a holomorphic function of $\bar{z}_{2}$ for $z_{2}$ in a suitable neighborhood of $z_{2}^{0}$. Hence it follows from (I) that

$$
\left(\overline{\partial w_{2} / \partial z_{2}}\right)^{-1}\left(w_{1}\left(z_{1}^{0}\right)+\bar{w}_{2}\right)
$$

is a holomorphic function of $\bar{z}_{2}$ in the same neighborhood. Since we may permit the point $z_{1}^{0}$ to vary over an open set, so that $w_{1}\left(z_{1}^{0}\right)$ varies over an open set, it follows moreover that both

$$
\left(\overline{\partial w_{2} / \partial z_{2}}\right)^{-1} \text { and }\left(\overline{\partial w_{2} / \partial z_{2}}\right)^{-1} \bar{w}_{2}
$$

are holomorphic functions of $\bar{z}_{2}$. Hence we have the following conclusion:

Lemma 1. The functions $(\partial w / \partial z)^{-1}$ and $(\partial w / \partial z)^{-1} w$, initially defined for $z \in T_{C_{1}}$, extend to be entire functions.

Now the function $|\partial w / \partial z|^{-1}$ does not vanish identically in $T_{C_{1}}$; hence it cannot vanish identically on the distinguished boundary of $T_{C_{1}}$. Since the affine automorphisms of $T_{C_{1}}$ are transitive on the distinguished boundary, we may suppose, after composing $w=f(z)$ with an affine map of form $z \rightarrow z+i b$, that $(\partial w / \partial z)^{-1}$ is nonsingular at $z=0$. Whence it follows from Lemma 1 above that $w=f(z)$ is holomorphic in a neighborhood of $z=0$. From the results of $\S I$, we know that $w(0)$ is a point of the distinguished boundary of $T_{C_{2}}$, and composing again with an appropriate affine map as above, we may assume that $w(0)=0$. Hence

LEMMA 2. After composing fore and aft with affine automorphisms of form $z \rightarrow z+i b$, we may suppose that $w=f(z)$ is defined in the neighborhood of $z=0$, that $w(0)=0$, and that $\partial w / \partial z$ is nonsingular at $z=0$.

So let us expand $w(z)$ at $z=0$ in a power series as follows:

$$
w^{v}(z)=w^{v}\left(z^{1}, z^{2}, \ldots, z^{m}\right)=\sum_{\mu} A_{\mu}^{v} z^{\mu}+\frac{i}{2} \sum_{r, s} U_{r, s}^{v} z^{r} z^{s}+\cdots
$$

where $U_{r, s}^{v}=U_{s, r}^{v}$.

Put more succinctly $w(z)=A z+(i / 2) U(z) z+\cdots$ where $A$ is a matrix whose $(\nu, \mu)$ entry is $A_{\mu}^{v}$ and $U(z)$ is a matrix whose $(\nu, s)$ entry in $\sum_{r} U_{r, s}^{v} z^{r}$. By its definition, $U\left(z_{1}\right) z_{2}=U\left(z_{2}\right) z_{1}$. It now follows that $\partial w / \partial z=A+i U(z)+\cdots$ and $(\partial w / \partial z)^{-1}$ $=A^{-1}-i A^{-1} U(z) A^{-1}+\cdots$.

We write as well for $z_{2}$ in a neighborhood of $0, B_{1}\left(z_{1}+\bar{z}_{2}\right)=B_{1}\left(z_{1}\right)+N\left(z_{1}, \bar{z}_{2}\right)$ $+\cdots$, where $N\left(z_{1}, z_{2}\right)$ is linear in $z_{2}$, and owing to homogeneity, $N\left(z_{1}, z_{1}\right)$ $=-2 B_{1}\left(z_{1}\right)$.

Now let us return to the function (I), which is independent of $z_{2}$. Taking power series expansion of the relevant parts, and sorting out the linear terms in $z_{2}$ (and still using homogeneity of the function $L_{1}$ ), we obtain

$$
N\left(z_{1}, \bar{z}_{2}\right) \bar{A}^{-1} w_{1}+i B_{1}\left(z_{1}\right) \bar{A}^{-1} \overline{U\left(z_{2}\right)} \bar{A}^{-1} w_{1}+2 B_{1}\left(z_{1}\right) \bar{z}_{2}=0 .
$$


In the last put $z_{2}=\bar{z}_{1}$. We know that $N\left(z_{1}, z_{1}\right)=-2 B_{1}\left(z_{1}\right)$. Furthermore, $B_{1}\left(z_{1}\right)$ is generally defined and nonsingular for $z_{1} \in T_{C_{1}}$, and is always nonsingular for $z_{1}$ real. Hence we may cancel $B_{1}\left(z_{1}\right)$ to obtain $\bar{A}^{-1} w-(i / 2) \bar{A}^{-1} \bar{U}(\bar{z}) \bar{A}^{-1} w=z$, which may be solved for $z$ near zero as $w=\left[I-(i / 2) \bar{U}(\bar{z}) \bar{A}^{-1}\right]^{-1} \bar{A} z$.

On comparing with the original expansion for $w(z)$, we conclude $A=\bar{A}, \bar{U}(\bar{z})$ $=U(z)$. We can say considerably more about the matrix $A$. For let $x \in C_{1}$ and let $\lambda$ be a positive real number. Then $\lim _{\lambda \rightarrow 0}(1 / \lambda) w(\lambda x)=A x$. Since $A$ is real, we conclude $A C_{1} \subset C_{2}$. We know $A$ is nonsingular, and if we reverse the roles of $\mathscr{D}_{1}$ and $\mathscr{D}_{2}$ and repeat the argument, we conclude $A^{-1} C_{2} \subset C_{1}$. Hence $C_{1}$ and $C_{2}$, thus also $\mathscr{D}_{1}$ and $\mathscr{D}_{2}$, are linearly equivalent. In summary,

THEOREM 3. Let $w$ be a biholomorphism of tube domain $\mathscr{D}_{1}$ onto tube domain $\mathscr{D}_{2}$. By composing $w$ fore and aft with translations of form $z \rightarrow z+i b$, we may suppose that $w$ is defined in a neighborhood of $z=0$, that $w(0)=0$, and that $A=\partial w(0) / \partial z$ is nonsingular. Then $A$ is real and gives a linear map of $C_{1}$ onto $C_{2}$ and the resulting $w$ may be written in the form $w=\left[I-(i / 2) U(z) A^{-1}\right]^{-1} A z$ for $z$ near zero. Moreover $\bar{U}(\bar{z})=U(z)$, and $U\left(z_{1}\right) z_{2}=U\left(z_{2}\right) z_{1}$.

Also if $w$ is a biholomorphism which is defined in a neighborhood of $0, w(0)=0$, and $A=\partial w(0) / \partial z$ is nonsingular, then $w$ is of form described above.

We now want to investigate in some detail what the possibilities for $U(z)$ are. Of course, if $U(z) \equiv 0$, then the map $w=f(z)$ is simply a linear map. So we will suppose $U \not \equiv 0$. If we take the map $w=f(z)$ and follow it by the map $z \rightarrow A^{-1} z$ of $\mathscr{D}_{2}$ onto $\mathscr{D}_{1}$, then we obtain an automorphism of $\mathscr{D}_{1}$, which we still denote $w$, of the form $w(z)=[I-(i / 2) U(z)]^{-1} z$ where the $U(z)$ appearing above differs slightly from the original, but still satisfies $U\left(z_{1}\right) z_{2}=U\left(z_{2}\right) z_{1}$.

Let $t \in \boldsymbol{R}^{m}$. Then the function $w_{t}(z)=w(z+i t)-w(i t)$ gives an automorphism of $\mathscr{D}_{1}$, which is defined and zero at 0 , and if $t$ is sufficiently close to zero, $w_{t}(z)$ will have a nonsingular Jacobian at $z=0$. A straightforward computation gives the Jacobian matrix as $J(t)$, where

$$
J(t)=\left[I+\frac{1}{2} U(t)\right]^{-1}\left[I-\frac{1}{2} U\left(\left[I+\frac{1}{2} U(t)\right]^{-1} t\right)\right] .
$$

From the last theorem we conclude

Lemma 4. For all real $t \in \boldsymbol{R}^{m}$ sufficiently close to zero, $J(t)$ belongs to the group of linear automorphisms of $C_{1}$.

Let $G$ be the group of linear automorphisms of $C_{1}$. It is known that $G$ is a closed subgroup of GL $(m, \boldsymbol{R})$. Let (s) be the Lie algebra of $G$.

Lemma 5. For all $t \in \boldsymbol{R}^{m}, U(t) \in \mathbb{S}$.

For the proof, let $\lambda$ be a real number confined to a neighborhood of zero. $J(\lambda t)$ is a curve in $\operatorname{GL}(m, R)$ which lies in $G$. The tangent to this curve at $\lambda=0$ is easily seen to be $-U(t)$. Since $G$ is a Lie subgroup it follows that $U(t) \in \mathbb{B}$. 
Now let $x$ and $y \in C^{m}$. We will define a commutative law of binary composition in $\boldsymbol{C}^{m}$ by $x \circ y=U(x) y$. If $x$ and $y \in \boldsymbol{R}^{m}$, then $x \circ y \in \boldsymbol{R}^{m}$, since $U(x)$ is real for real $x$. Define, for all natural numbers $b, z^{b}$ by $z^{b}=U^{b-1}(z) z$. In these terms, the power series for $w$ may be written as $w=\sum_{v=0}^{\infty}\left(i^{v} / 2^{v}\right) z^{v+1}$.

Now we return to the equation (II) of this section, or more precisely, to the analogue of (II) for the map $w$ of $\mathscr{D}_{1}$ to $\mathscr{D}_{1}$. The analogous equation is

$$
N\left(z_{1}, \bar{z}_{2}\right) w_{1}+i B_{1}\left(z_{1}\right) U\left(\bar{z}_{2}\right) w_{1}+2 B_{1}\left(z_{1}\right) \bar{z}_{2}=0 .
$$

$B_{1}\left(z_{1}\right)$ is homogeneous of weight -2 , whence $N\left(z_{1}, \bar{z}_{2}\right)$ is homogeneous of weight -3 in the variable $z_{1}$. Also $N\left(z_{1}, \bar{z}_{2}\right) w_{1}=N\left(z_{1}, w_{1}\right) \bar{z}_{2}$. So from the last we obtain $N(z, w)+i B_{1}(z) U(w)+2 B_{1}(z)=0$, which is valid for $z$ such that $L_{1}(z) \neq 0$, hence valid in particular for $z$ real. If we use the power series expansion of $w$ from above in the last equation, and sort out the homogeneous part of weight $\nu-1$, we obtain

$$
\frac{1}{2} N\left(z, z^{v+2}\right)+B_{1}(z) U\left(z^{v+1}\right)=0 \text { for } \nu=0,1,2, \ldots
$$

Now let $z=x$ be real, so $x \in C_{1}$. Then $B_{1}(x)$ is defined, and so is $N(x, w)$. From its definition, it follows that the matrix $N(x, w)$ is symmetric, since $B_{1}(x)$ is symmetric. Thus

LEMMA 6. For $x \in C_{1}$, and all $\nu \geqq 0, B_{1}(x) U\left(x^{\nu+1}\right)$ is symmetric.

Using the last we shall be able to show that the binary law of composition defined above gives a Jordan algebra; i.e., we show that $x \circ\left(y \circ x^{2}\right)=x^{2} \circ(y \circ x)$. This is the same as showing that the matrices $U(x)$ and $U\left(x^{2}\right)$ commute. For most of the references to Jordan algebras, we direct the reader to [5].

The composition $w(w(z))$ of $w$ with itself is an automorphism of $\mathscr{D}_{1}$, defined and zero at zero, with Jacobian at the origin being the identity matrix. Hence by Theorem 3 , there exists uniquely a matrix $V(z)$, linear in $z$, with $V\left(z_{1}\right) z_{2}=V\left(z_{2}\right) z_{1}$, such that $w(w(z))=[I-(i / 2) V(z)]^{-1} z$.

By direct examination, we see immediately that the quadratic part of $w(w(z))$ is just $i U(z) z$. Thus $V(z)=U(2 z)$, and so $w(w(z))=[I-i U(z)]^{-1} z$. Hence the homogeneous part of weight 4 in the series expansion of $w(w(z))$ is just $i^{3} z^{4}$.

On the other hand, direct computation of the composition of $w$ with itself gives for the homogeneous part of weight 4 the following expression:

$$
\frac{3}{4} i^{3} z^{4}+\frac{1}{4} i^{3} z^{2} \circ z^{2},
$$

so we must have $z^{2} \circ z^{2}=z^{4}$. Now we polarize the last identity as follows. Replace $z$ by $x+y$, and sort out the linear part in $y$. We obtain

$$
4 U\left(x^{2}\right) U(x) y=2 U^{3}(x) y+U\left(x^{3}\right) y+U(x) U\left(x^{2}\right) y
$$

which says, of course, that

$$
4 U\left(x^{2}\right) U(x)=2 U^{3}(x)+U\left(x^{3}\right)+U(x) U\left(x^{2}\right) .
$$


Let $x \in C_{1}$. Then by Lemma $6, U\left(x^{\nu}\right)(\nu \geqq 1)$ is always selfadjoint with respect to the nondegenerate symmetric bilinear form $\left\langle B_{1}(x) \cdot, \cdot\right\rangle$. By taking adjoints of the last equation with respect to this form, we obtain

$$
4 U(x) U\left(x^{2}\right)=2 U^{3}(x)+U\left(x^{2}\right) U(x)+U\left(x^{3}\right),
$$

and comparing the last two equations, we conclude that $U(x)$ and $U\left(x^{2}\right)$ commute for $x \in C$, so commute always. Hence

THEOREM 7. The law of composition $x \circ y=U(x) y, x, y \in \boldsymbol{R}^{m}$, defines a Jordan algebra structure on $\boldsymbol{R}^{m}$. For any $t \in \boldsymbol{R}^{m}, U(t)$ belongs to the Lie algebra of the group of linear automorphism of $C_{1}$.

In the next section we shall show, among other things, that if the conclusions of Theorem 7 hold, then the map $w(z)=[I-(i / 2) U(z)]^{-1} z$ initially defined in a neighborhood of the origin, extends to give an automorphism of $\mathscr{D}_{1}=T_{C_{1}}$. This result, taken in conjunction with Theorem 3 , gives, modulo information about the group of linear automorphisms of $C_{1}$, reasonably complete and precise information about the full group of automorphisms of $\mathscr{D}_{1}$, or about biholomorphisms from $\mathscr{D}_{1}$ onto a second tube domain $\mathscr{D}_{2}$.

We shall also give a geometric characterization of cones $C_{1}$ for which the conclusions of Theorem 7 hold.

IV. Cones with Jordan algebra structure. We suppose now that we have the following situation. $C$ is a regular open convex cone in $\boldsymbol{R}^{m}$, equipped with usual scalar product $\langle\cdot, \cdot\rangle$, which we frequently extend by linearity to the complexification $\boldsymbol{C}^{m}$ of $\boldsymbol{R}^{m}$. Furthermore, we suppose that $\boldsymbol{R}^{m}$ may be given the Jordan algebra structure $\mathscr{A}$, such that the endomorphism $U(x): y \rightarrow U(x) y=x \circ y$ is for all $x \in \boldsymbol{R}^{m}$ an element of the Lie algebra (S) of the group $G$ of automorphisms of $C$. We shall suppose $\mathscr{A}$ is nontrivial, that is we do not always have $x \circ y=0$. Under these conditions, we shall completely analyze the structure of $C$ and the Jordan algebra $\mathscr{A}$.

Conforming to the notation introduced earlier, but with subscripts 1 and 2 to distinguish between $C_{1}$ and $C_{2}$ now gone, let $L(z+\bar{w})$ be kernel function for $T_{C}$. We shall consider $L$ only for real values of the argument.

As a matter of fact, as far as the analysis of this section is concerned, the function $L$ could be replaced with the Koecher norm function $M$ introduced incidentally in Lemma 2 of $§ I$, which enjoys most of the same useful properties as $L$. But since nothing much is gained (or lost) by such substitution, we will confine ourselves to the functions we have already systematically introduced.

Let

$$
b(x)=\partial \log L(x) / \partial x \quad \text { and } \quad B(x)=\partial^{2} \log L(x) / \partial x \partial x,
$$

all functions defined for $x \in C$. 
Let $g \in G$. From the law of transformation of kernel function, we have $L(g x)|g|^{2}$ $=L(x)$.

Let $\mathfrak{g} \in(\mathfrak{S}$. Then $\exp \lambda \mathfrak{g} \in G$. Differentiating the last equality with respect to $\lambda$, and then setting $\lambda=0$, we obtain

$$
\langle b(x), \mathfrak{g} x\rangle=-2 \operatorname{tr} \mathfrak{g},
$$

and differentiating last with respect to $x$, we obtain

$$
B(x) \mathfrak{g} x=-{ }^{t} \mathfrak{g} b(x)={ }^{t} \mathfrak{g} B(x) x
$$

where for the second equality, we have used the additional fact, stemming from the homogeneity of $L(x)$, that $B(x) x=-b(x)$.

The equations (I) and (II) are the basis of our analysis of $\mathscr{A}$.

LeMma 1. $B(x) U(x)={ }^{t} U(x) B(x)$ for $x \in C$.

Since $U(s) \in$ SS for any $s \in R^{m}$, we have from (II) above that $B(x) U(s) x$ $=-{ }^{t} U(s) b(x)$. Let $t$ be arbitrary in $\boldsymbol{R}^{m}$. Then

$$
\langle B(x) U(s) x, t\rangle=-\left\langle{ }^{t} U(s) b(x), t\right\rangle \text { or }\langle B(x) U(x) s, t\rangle=-\langle b(x), s \circ t\rangle .
$$

Since the right-hand side of the last equality is symmetric in $s$ and $t$, we conclude that $B(x) U(x)$ is symmetric.

There exists a unique $d \in \boldsymbol{R}^{m}$ such that $\operatorname{tr} U(s)=\langle s, d\rangle$. Then we have

LEMMA 2. ${ }^{t} U(x) b(x)=-2 d$ for $x \in C$.

We have $\langle b(x), U(s) x\rangle=\langle b(x), U(x) s\rangle=\left\langle{ }^{t} U(x) b(x), s\right\rangle=-2 \operatorname{tr} U(s)=-2\langle s, d\rangle$, which gives desired result.

Associated with every Jordan algebra $\mathscr{A}$, there is a symmetric bilinear form $R(\cdot, \cdot)$, called trace form, defined by $R(x, y)=\operatorname{tr} U(x \circ y)$. If $R$ is nondegenerate, then $\mathscr{A}$ is called semisimple. The set $\mathscr{R}$ of $x \in \mathscr{A}$ such that $R(x, y) \equiv 0$ for all $y \in \mathscr{A}$ is called the radical of $\mathscr{A}$, and is an ideal of $\mathscr{A}$. The endomorphisms $U(x)$ are selfadjoint with respect to the trace form. We define the kernel $\mathscr{K}$ of $\mathscr{A}$ to be the set of $x \in \mathscr{A}$ such that $U(x)=0$. Clearly $\mathscr{K} \subset \mathscr{R}$, and our assumption of nontriviality of $\mathscr{A}$ implies that $\mathscr{K} \nsubseteq \mathscr{A}$.

There exists a unique matrix $R$, necessarily symmetric, such that $R(x, y)$ $=\langle R x, y\rangle$. But

$$
R(x, y)=\operatorname{tr} U(x \circ y)=\langle x \circ y, d\rangle=\langle U(x) y, d\rangle=\left\langle{ }^{t} U(x) d, y\right\rangle=\langle R x, y\rangle .
$$

Hence

LEMMA 3. ${ }^{t} U(x) d=R x$.

But more significantly, we have

THEOREM 4. For any $x \in C, 2 R=B(x)\left\{2 U^{2}(x)-U\left(x^{2}\right)\right\}$.

For the purpose of the proof, define $P(x)$ by $P(x)=2 U^{2}(x)-U\left(x^{2}\right)$; also, let $\left[\mathfrak{g}_{1}, \mathfrak{g}_{2}\right]=\mathfrak{g}_{1} \mathfrak{g}_{2}-\mathfrak{g}_{2} \mathfrak{g}_{1}$ be the commutator of the endomorphisms $\mathfrak{g}_{1}$ and $\mathfrak{g}_{2}$. 
Let $u, v \in \boldsymbol{R}^{m}, x \in C$, and put $\mathfrak{g}=U(x \circ v)+[U(x), U(v)]$. Clearly $\mathfrak{g} \in \mathfrak{S S}$, and as a trivial computation shows $\mathfrak{g} x=P(x) v$ and $\mathfrak{g} u=\tilde{\mathrm{g}} x$ where $\tilde{\mathrm{g}}=U(u \circ v)+[U(u), U(v)]$ $\in$ (S). Now using (I) and (II),

$$
\begin{aligned}
\langle u, B(x) P(x) v\rangle & =\langle u, B(x) \mathfrak{g} x\rangle=-\left\langle u,{ }^{t} \mathfrak{g} b(x)\right\rangle \\
& =-\langle\mathfrak{g} u, b(x)\rangle=-\langle\tilde{\mathfrak{g}} x, b(x)\rangle=2 \operatorname{tr} \tilde{\mathfrak{g}} \\
& =2 \operatorname{tr} U(u \circ v)=2\langle u, R v\rangle
\end{aligned}
$$

whence the statement of the theorem.

Corollary 5. For any positive integer $\nu$, and $x \in C, B(x) U\left(x^{v}\right)={ }^{t} U\left(x^{v}\right) B(x)$.

The statement is true for $\nu=1$, by Lemma 1 . Its truth for $\nu=2$ then follows from the last theorem, since $R$ is symmetric. But in any Jordan algebra, $U\left(x^{\nu}\right)$ is a polynomial in the commuting endomorphisms $U(x)$ and $U\left(x^{2}\right)$, which yields the general statement.

The theorem above has some other important consequences, which we give now for convenience.

THEOREM 6. Let $r, s \in \mathscr{R}$. Then $r \circ s \in \mathscr{K}$ and $U(r) U(s)=U(s) U(r)$.

Let $x$ be arbitrary and $r \in \mathscr{R}$. Then $U(r) U(x)-U(x) U(r)=U(r \circ x)$.

For the proof, let $r \in \mathscr{R}$. Then $R r=0$, and since $B(x)$ is nonsingular for $x \in C$, $\left\{2 U^{2}(x)-U\left(x^{2}\right)\right\} r=0$ for all $x \in C$. Since this is just a polynomial identity, holding for $x$ in an open set, it holds for all $x$. Replacing $x$ by $x+y$, and picking out the part linear in $y$, we obtain

$$
\{U(x) U(y)+U(y) U(x)-U(x \circ y)\} r=0
$$

identically in $x$ and $y$, which gives $U(r \circ x)=U(r) U(x)-U(x) U(r)$, one of the statements of the theorem. If in the last $x=s \in \mathscr{R}$, then since the right-hand side changes sign if $r$ and $s$ are interchanged, and the left-hand side remains unchanged, it follows that $U(r)$ and $U(s)$ commute and that $U(r \circ s)=0$; i.e., $r \circ s \in \mathscr{K}$.

The equation $U(x \circ y) r=U(x) U(y) r+U(y) U(x) r$ appearing above has a significance which we will exploit later. For the moment, suffice it to say that the equation indicates that the map $\left.x \rightarrow U(x)\right|_{\mathscr{R}}$, where $\left.U(x)\right|_{\mathscr{R}}$ is the endomorphism $U(x)$ restricted to the invariant subspace $\mathscr{R}$, is a representation of the Jordan algebra $\mathscr{A}$ in End $\mathscr{R}$.

As an immediate consequence of the fact that $\mathscr{R}^{2} \subset \mathscr{K}$, we have

COROLlaRY 7. For $r, s \in \mathscr{R}$, and $x$ arbitrary $U(x) U(r) U(s)=0$.

Also,

COROllary 8. Let $r \in \mathscr{R}$. The map $x \rightarrow U(r) x$ is a derivation of $\mathscr{A}$.

We must verify that $U(r)(x \circ y)=(U(r) x) \circ y+x \circ U(r) y$. But this follows immediately from the statement of the last theorem that $U(r) U(x)-U(x) U(r)$ $=U(r \circ x)$. 
The endomorphism exp $U(r)$ is, of course, a linear automorphism of $C$. But from the last corollary we have the important additional fact:

TheOREM 9. For $r \in \mathscr{R}$, the endomorphism $\exp U(r)$ is an automorphism of $\mathscr{A}$.

This result is, of course, simply a formal consequence of the fact that the map $x \rightarrow U(r) x$ is a derivation.

Now if $r \in \mathscr{R}$, then it is known that both $r$ and $U(r)$ are nilpotent. In particular $\operatorname{tr} U(r)=\langle r, d\rangle=0$. It follows that $d$ is in the range space of $R$; i.e., there exists a vector $c$ such that $R c=d$. Actually we can show

LEMMA 10. There exists an idempotent e such that $R e=d$.

Let $R c=d$. Then ${ }^{t} U(x) R c={ }^{t} U(x) d=R U(x) c=R U(c) x=R x$. Hence $R U(c)=R$, whence $R c^{\nu}=R c=d$ for all positive integers $\nu$.

If $c$ were nilpotent then $d=0$. Whence $R x={ }^{t} U(x) d=0$, implying $R \equiv 0$, implying $\mathscr{R}=\mathscr{A}$. This implies $U(x)$ is nilpotent for all $x \in \mathscr{A}$, in particular for $x \in C$. But by Lemma $1, U(x)$ is semisimple, hence $U(x)=0$ for $x \in C$. But this implies $U(x) \equiv 0$ for all $x \in \mathscr{A}$, which contradicts our assumption of nontriviality of $\mathscr{A}$.

Thus $c$ is not nilpotent. Hence the commutative and associative algebra generated by $c$ contains an idempotent $e . e=\sum_{v \geqq 1} a_{\nu} c^{\nu}$, and $e \circ c^{l}=c^{l}$ for all sufficiently large $l$. Then $\operatorname{Re}=\sum_{\nu} a_{v} R c^{\nu}=d \sum a_{v}=\alpha d$, where we have put $\alpha=\sum a_{v}$. Repeating the argument used above, we obtain $R U(e)=\alpha R$, so $R U(e) c^{l}=\alpha R c^{l}=\alpha d=R c^{l}=d$. Hence $\alpha=1$, and we are done.

LEMMA 11. Let $e^{\prime}$ be a second idempotent such that $R^{\prime}=d$. Then there exists an $r \in \mathscr{R}$ such that $e^{\prime}=(\exp U(r)) e$.

Since $R\left(e^{\prime}-e\right)=0, e^{\prime}=e+s$ where $s \in \mathscr{R}$. Then $e^{\prime}=e^{\prime 2}=e^{2}+2 e \circ s+s^{2}$, so $s$ $=2 e \circ s+s^{2}$, whence $s^{2}=\left(2 e \circ s+s^{2}\right)^{2}=4(e \circ s)^{2}=4 U(e \circ s) U(e) s$, using the fact that $s^{2} \in \mathscr{K}$. Now, using Theorem 6, we obtain that

$$
\begin{aligned}
s^{2} & =4[U(s) U(e)-U(e) U(s)] U(e) s=4 U(s) U^{2}(e) s \\
& =2 U(s) U\left(e^{2}\right) s=2 U^{2}(s) e,
\end{aligned}
$$

where we have used additionally the fact that $\mathscr{R}^{2} \subset \mathscr{K}$ to conclude that $U(e) U(s) U(e) s=0$, and the fact that $\left\{2 U^{2}(e)-U\left(e^{2}\right)\right\} s=0$, following from text of Theorem 6.

Finally, $e^{\prime}=e+2 e \circ s+s^{2}=e+2 e \circ s+2 U^{2}(s) e=\{\exp U(2 s)\} e$, since $U^{3}(s)=0$ by Corollary 7 .

LEMMA 12. Let $c$ be any vector such that $R c=d$. Then the null space of $2 U^{2}(c)$ $-U\left(c^{2}\right)$ is exactly $\mathscr{R}$.

Let $R c=d$. Then by an argument used earlier, $R U(c)=R$, whence $R U^{2}(c)=R$. Also then, $R c^{2}=d$, so repeating argument, $R U\left(c^{2}\right)=R$. Hence, $R\left\{2 U^{2}(c)-U\left(c^{2}\right)\right\}$ $=R$. We already know that the null space of $2 U^{2}(x)-U\left(x^{2}\right)$ contains the radical for 
any $x \in \mathscr{A}$. But then the last equation shows that the null space of $2 U^{2}(c)-U\left(c^{2}\right)$ is precisely the radical, completing the proof.

Now let $e$ be an idempotent such that $R e=d$. With respect to $e$, we will take a Pierce decomposition of $\mathscr{A}, \mathscr{A}=\mathscr{A}_{1} \oplus \mathscr{A}_{1 / 2} \oplus \mathscr{A}_{0}$. If we want to indicate the dependence of the decomposition on $e$, we will write $\mathscr{A}=\mathscr{A}_{1}(e) \oplus \mathscr{A}_{1 / 2}(e) \oplus \mathscr{A}_{0}(e)$. $\mathscr{A}_{\rho}(e)$ is characterized by $e \circ a_{\rho}=\rho a_{\rho}$ if $a_{\rho} \in \mathscr{A}_{\rho}$. As a general property of the Pierce decomposition, it is known that

$$
\begin{array}{rlrl}
\mathscr{A}_{0}^{2} & \subset \mathscr{A}_{0}, & \mathscr{A}_{0} \mathscr{A}_{1 / 2} \subset \mathscr{A}_{1 / 2}, & \mathscr{A}_{0} \mathscr{A}_{1}=\{0\}, \\
\mathscr{A}_{1 / 2}^{2} \subset \mathscr{A}_{0}+\mathscr{A}_{1}, & \mathscr{A}_{1 / 2} \mathscr{A}_{1} \subset \mathscr{A}_{1 / 2}, & \mathscr{A}_{1}^{2} \subset \mathscr{A}_{1} .
\end{array}
$$

Thanks to the geometric backdrop of the present situation, we can improve these inclusions considerably.

THEOREM 13. $\mathscr{A}_{0}=\mathscr{K}, \mathscr{A}_{0}+\mathscr{A}_{1 / 2}=\mathscr{R}$.

The second statement is immediate, since $\mathscr{R}$ is precisely the null space of $2 U^{2}(e)-U\left(e^{2}\right)=2 U^{2}(e)-U(e)$.

As for the first, obviously $\mathscr{K} \subset \mathscr{A}_{0}$. Since we know $\mathscr{R}^{2} \subset \mathscr{K}$, the inclusions $\mathscr{A}_{0} \mathscr{A}_{1 / 2} \subset \mathscr{A}_{1 / 2}, \mathscr{A}_{1 / 2}^{2} \subset \mathscr{A}_{0}+\mathscr{A}_{1}$, and $\mathscr{A}_{0}^{2} \subset \mathscr{A}_{0}$ may be sharpened respectively to $\mathscr{A}_{0} \mathscr{A}_{1 / 2}=\{0\}, \mathscr{A}_{1 / 2}^{2} \subset \mathscr{A}_{0}$, and $\mathscr{A}_{0}^{2} \subset \mathscr{K}$.

Now let $a_{0} \in \mathscr{A}_{0}$. I claim that $U^{2}\left(a_{0}\right)=0$. By virtue of the inclusions $\mathscr{A}_{0} \mathscr{A}_{1 / 2}=\{0\}$ and $\mathscr{A}_{0} \mathscr{A}_{1}=\{0\}$, it is clear that $U^{2}\left(a_{0}\right)\left(\mathscr{A}_{1 / 2} \oplus \mathscr{A}_{1}\right)=0$. Since $\mathscr{A}_{0}^{2} \subset \mathscr{K}, U\left(a_{0}^{2}\right) \mathscr{A}=0$. If $r$ is arbitrary in $\mathscr{R}$, then $2 U^{2}\left(a_{0}\right) r=U\left(a_{0}^{2}\right) r=0$. Hence $U^{2}\left(a_{0}\right) \mathscr{A}_{0}=0$, which proves the claim.

Now let $x$ be arbitrary in $C$, and $\lambda$ any real number. Then exp $\lambda U\left(a_{0}\right)=1+\lambda U\left(a_{0}\right)$ is an automorphism of $C$. Hence $\exp \left(\lambda U\left(a_{0}\right)\right) x=x+\lambda U\left(a_{0}\right) x \in C$ for any real $\lambda$. This contradicts the assumption that $C$ does not contain an entire straight line, unless $U\left(a_{0}\right) x=0$. Since the last must be true for $x \in C$, it must be true for arbitrary $x$. Hence $U\left(a_{0}\right)=0$, which implies $a_{0} \in \mathscr{K}$, completing the proof.

There is another conclusion, which will be useful later.

LemMA 14. If $\mathscr{K}=\{0\}$, then $\mathscr{R}=\{0\}$, and $\mathscr{A}$ is semisimple.

For suppose $\mathscr{K}=\{0\}$. Then since $\mathscr{R}^{2} \subset \mathscr{K}, \mathscr{R}^{2}=\{0\}$. Let $r \in \mathscr{R}$. Then $U^{2}(r)=0$, for $U^{2}(r) x$ is the product of two elements of $\mathscr{R}$. The same geometrical argument used in the last lemma now enables us to conclude that $U(r)=0$; i.e., $r \in \mathscr{K}$; i.e., $r=0$, completing the proof.

As our next task, we will investigate the case of semisimple $\mathscr{A}$.

THEOREM 15. If $\mathscr{A}$ is semisimple, then $C$ is a linearly homogeneous, selfadjoint convex cone.

For suppose $\mathscr{A}$ semisimple. Then $R$ is nonsingular. Let $c \in C$. I claim that the linear space spanned by vectors of form $U(x) c$ and $\{U(x) U(y)-U(y) U(x)\} c, x$ and $y$ being arbitrary in $\mathscr{A}$, is all of $\mathscr{A}$. Suppose to the contrary that there exists a $v$ such that $\langle R v, U(x) c\rangle=0$ and $\langle R v, U(x) U(y) c-U(y) U(x) c\rangle=0$. 
Since $U(x)$ is selfadjoint with respect to the trace form, the first implies $\langle R U(c) v, x\rangle=0$, so $U(c) v=0$. From the second, we obtain that

$$
\langle R U(c) U(x) v-R U(x \circ c) v, y\rangle=0
$$

whence $U(c) U(x) v=U(x \circ c) v$, so $U(c) U(v)=U(v) U(c)$. Hence $0=U(c) U(c) v$ $=U(c) U(v) c=U(v) c^{2}$, or $U\left(c^{2}\right) v=0$. Thus $\left\{2 U^{2}(c)-U\left(c^{2}\right)\right\} v=0$. But for $c \in C$, since $2 R=B(c)\left\{2 U^{2}(c)-U\left(c^{2}\right)\right\}, 2 U^{2}(c)-U\left(c^{2}\right)$ is nonsingular, whence $v=0$, proving the claim.

Consider now the Lie algebra $\mathfrak{S}$, generated by $U(x), x \in \mathscr{A}$, which contains, of course, the commutators $U(x) U(y)-U(y) U(x)$. This is the Lie algebra of some analytic group $H$ of automorphisms of $C$. For $c \in C, \mathfrak{h} c$ spans the tangent space to $C$ at $c$, as $\mathfrak{h}$ varies over $\mathfrak{S}$. It follows that $H$ is transitive on $C$, i.e., $C$ is linearly homogeneous. On the other hand, it is well known that the endomorphisms $U(x)$ of a semisimple Jordan algebra are a completely reducible family. Hence $H$ is completely reducible over the reals. By a minor modification of Theorem 1.5 of [7], it now follows that $C$ is selfadjoint.

As the referee has pointed out, it suffices in Theorem 1.5 to assume only that $\mathscr{A}$ has a unit, as it then follows from Lemma 14 that $\mathscr{K}=\{0\}$, so $\mathscr{A}$ is semisimple.

The ambient Euclidean space for a selfadjoint linearly homogeneous cone already comes equipped with the structure of a semisimple Jordan algebra $\mathscr{A}^{\prime}$, satisfying the hypothesis of this section. Let $V(x)$ be the endomorphism giving multiplication in $\mathscr{A}^{\prime}$, and let $R^{\prime}$ be the matrix associated with the trace form in $\mathscr{A}^{\prime}$.

THEOREM 16. The multiplications $U(x)$ and $V(x)$ are each "mutants" of the other.

We know $2 R=B(x)\left\{2 U^{2}(x)-U(U(x) x)\right\}$ and $U(e) x=x$, since $\mathscr{A}_{1}=\mathscr{A}$. Also $2 R^{\prime}=B(x)\left\{2 V^{2}(x)-V(V(x))\right\}$. Hence

$$
\left\{2 V^{2}(x)-V(V(x) x)\right\} R^{\prime-1} R=\left\{2 U^{2}(x)-U(U(x) x)\right\} .
$$

Let $R^{\prime-1} R e=f$. Then $2 V^{2}(x) f-V(V(x) x) f=2 U(x) x$. Replace $x$ by $x+y$, and sort out linear terms in $y$, to obtain

$$
U(x)=V(x) V(f)-V(f) V(x)+V(V(x) f) .
$$

By definition, the last says $V(x)$ is a mutant of $U(x)$. An analogous computation gives the dependence of $V(x)$ on $U(x)$.

It will be useful to compute now what the nonlinear map $w(z)=[I-(i / 2) U(z)]^{-1} z$ is in the case of semisimple $\mathscr{A}$ currently at hand.

The formula above for $w$ is, of course, not necessarily defined for all $z$. It is certainly defined for small $z$. Now $T_{C}$ has a holomorphic symmetry $z \rightarrow z^{*}$, which may be computed everywhere in $T_{C}$ by the formula $z^{*}=P^{-1}(z) z$, where $P(z)$ $=2 V^{2}(z)-V(V(z) z)$ [5]. From a known formula [5],

$$
2 U^{2}(z)-U(U(z) z)=\left\{2 V^{2}(z)-V(V(z) z)\right\}\left\{2 V^{2}(f)-V(V(f) f)\right\},
$$


and from the proof of Theorem 16, we see that $2 V^{2}(f)-V(V(f) f)=R^{\prime-1} R$ and so is nonsingular. Since $U(e)=I$,

$$
w(z-2 i e)=2 i U^{-1}(z)(z-2 i e)=2 i e+4 U^{-1}(z) e,
$$

since $U(z) e=z$, so $U^{-1}(z) z=e$. For almost all $z$,

$$
\begin{aligned}
U^{-1}(z) e & =\left[2 U^{2}(z)-U(U(z) z)\right]^{-1} z=\left[2 V^{2}(f)-V(V(f) f)\right]^{-1} P^{-1}(z) z \\
& =\left[2 V^{2}(f)-V(V(f) f)\right]^{-1} z^{*}
\end{aligned}
$$

Let $A^{-1}=2 V^{2}(f)-V(V(f) f)$. $A$ is known to be an automorphism of $C$. Hence, $w(z)=4 A(z+2 i e)^{*}+2 i e$ which is a formula for $w(z)$ clearly showing it gives an automorphism of $T_{C}$.

We return now to the general case of nonsemisimple $\mathscr{A}, e$ being an idempotent such that $\operatorname{Re}=d$, and $\mathscr{A}=\mathscr{A}_{1}+\mathscr{A}_{1 / 2}+\mathscr{A}_{0}$ the associated Pierce decomposition. Let $\pi_{1}, \pi_{1 / 2}, \pi_{0}$ be the corresponding projections.

THEOREM 17. $C_{0}=\pi_{0} C$ and $C_{1}=\pi_{1} C$ are regular convex cones lying in the closure $\bar{C}$ of $C$.

Moreover $C_{1}$ is a homogeneous selfadjoint cone.

Let $x \in C$, and write $x=x_{1}+x_{1 / 2}+x_{0}$. For any real $\lambda$, exp $\lambda U(e)$ is an automorphism of $C$. Hence $\exp (\lambda U(e)) x=x_{0}+(\exp (\lambda / 2)) x_{1 / 2}+(\exp \lambda) x_{1} \in C$. Let $\lambda$ go to $-\infty$ to conclude $x_{0} \in \bar{C}$. Also

$$
\exp (-\lambda) \exp (\lambda U(e)) x=\exp (-\lambda) x_{0}+\exp (-\lambda / 2) x_{1 / 2}+x_{1} \in C .
$$

Let $\lambda$ go to $+\infty$ to conclude $x_{1} \in \bar{C}$. Since $\bar{C}$ does not contain an entire straight line, $C_{0}$ and $C_{1}$ are regular. They are clearly relatively open in $\mathscr{A}_{0}$ and $\mathscr{A}_{1}$ respectively, since projection is an open map.

$C_{1}$ is a cone with Jordan structure $\mathscr{A}_{1}$. Since $\mathscr{A}_{1}$ has a unit, $C_{1}$ is homogeneous selfadjoint.

Using the last and some earlier results, we can draw a rather interesting conclusion.

THEOREM 18. Let $C$ be an arbitrary regular convex cone. Suppose the geodesic symmetry at one point of $T_{C}$ extends to a holomorphic map of $T_{C}$. Then $C$ is homogeneous selfadjoint and $T_{C}$ is Hermitian symmetric.

First, we want to note that the geodesic symmetry cannot be an affine map. For suppose it were, then it must be of the form $w=A z+i t, t \in \boldsymbol{R}^{m}$, and $A$ an automorphism of $C$ such that $A^{2}=I$ and $A t=-t$. Let $z_{0}$ be the fixed point. We cannot have $A=-I$, since $A$ is an automorphism of $C$. Hence there exists nonzero $x \in \boldsymbol{R}^{m}$ such that $A x=x$. But then for any complex $\lambda, w\left(z_{0}+\lambda x\right)=z_{0}+\lambda x$, which contradicts the fact that $z_{0}$ is an isolated fixed point.

Up to conjugation by an imaginary shift, we have by Theorem 3 of §III that $w$ is of the form $w(z)=A[I-(i / 2) U(z)]^{-1} z+i t$ where $t \in \boldsymbol{R}^{m} . A$ is an automorphism of 
$C$ and $U(z)$ is not identically 0 . Let $\mathscr{K}$ be the kernel of the associated Jordan algebra, and let $k \in \mathscr{K}$. It is easy to see that $w(z+k)=w(z)+A k$. Replace $z$ by $w(z)$, and use the fact that $w$ is an involution to obtain $w(z+A k)=w(z)+k$. Hence $w(z+k+A k)=w(z)+k+A k$. Let $z_{0}$ be the fixed point of $w$. Then $w\left(z_{0}+k+A k\right)$ $=z_{0}+k+A$, which contradicts the assumption that $z_{0}$ is isolated, unless $k+A k=0$; i.e., $A k=-k$ for all sufficiently small $k \in \mathscr{K}$, and so for all of $\mathscr{K}$.

Now let $k \in C_{0}$. Then $k \in \bar{C}$. $A$ is an automorphism of $C$. Hence $-k=A k \in \bar{C}$. This contradicts the regularity of $C$, unless $\mathscr{K}=\{0\}$, which implies the Jordan algebra is semisimple; hence $C$ is homogeneous selfadjoint. But then it is known [7] that $T_{C}$ is Hermitian symmetric, completing the proof.

Let $x \in C$. Consider the commutative associative algebra $\mathscr{A}_{x}$ generated by $x^{2}, x^{3}, \ldots$

\section{LEMMA $19 . \mathscr{A}_{x}$ is a semisimple associative algebra.}

For suppose an element $u=a_{2} x^{2}+a_{3} x^{3}+\cdots+a_{r} x^{r}$ is nilpotent. Then $U(u)$ is nilpotent. But we know $U(u)$ is semisimple, since $B(x) U(u)={ }^{t} U(u) B(x)$, so we must have $U(u)=0$. Hence $u \in \mathscr{K}$. Hence $0=U(u) x=a_{2} x^{3}+a_{3} x^{4}+\cdots+a_{r} x^{r+1}$. Now $2 R=B(x)\left\{2 U^{2}(x)-U\left(x^{2}\right)\right\}$, whence for $\nu \geqq 0,2 R x^{\nu}=B(x) x^{\nu+2}$. Thus

$$
R\left(a_{2} x+a_{3} x^{2}+\cdots+a_{r} x^{r-1}\right)=\frac{1}{2} B(x)\left(a_{2} x^{3}+a_{3} x^{4}+\cdots+a_{r} x^{r+1}\right)=0 .
$$

So $a_{2} x+a_{3} x^{2}+\cdots+a_{r} x^{r-1} \in \mathscr{R}$. This implies that $a_{2} x+a_{3} x^{2}+\cdots+a_{r} x^{r-1}$ is nilpotent, so $U\left(a_{2} x+a_{3} x^{2}+\cdots+a_{r} x^{r-1}\right)$ is nilpotent, and the argument used above shows that $a_{2} x+a_{3} x^{2}+\cdots+a_{r} x^{r-1} \in \mathscr{K}$. Hence $u=\left(a_{2} x+a_{3} x^{2}+\cdots+a_{r} x^{r-1}\right) \circ x$ $=0$, completing the proof.

We let $e_{x}$ be the unit for $\mathscr{A}_{x} . e_{x}$ is a polynomial without constant or linear term in $x$.

Corollary 20. $R e_{x}=d$.

$U(x)$ and $U\left(e_{x}\right)$ commute. Hence $U^{2}(x) e_{x}=U(x) U\left(e_{x}\right) x=U\left(e_{x}\right) x^{2}=x^{2}$. Also $U\left(x^{2}\right) e_{x}=x^{2}$. Hence

$$
2 R e_{x}=B(x)\left\{2 U^{2}(x)-U\left(x^{2}\right)\right\} e_{x}=B(x) x^{2}=B(x) U(x) x=-{ }^{t} U(x) b(x)=2 d,
$$

which gives desired conclusion.

Now let $x \in C$. Since $e_{x}$ is a polynomial in $x$, it follows that $e_{x} \circ\left(e_{x} \circ x\right)=e_{x} \circ x$ and so $e_{x} \circ\left(x-e_{x} \circ x\right)=0$. Hence $e_{x} x \in \mathscr{A}_{1}\left(e_{x}\right)$, and $x-e_{x} \circ x \in \mathscr{A}_{0}\left(e_{x}\right)$. So we may write $x=x_{1}+x_{0}, x_{1} \in \mathscr{A}_{1}\left(e_{x}\right)$ and $x_{0} \in \mathscr{K}$. We already know that $x_{0}$ and $x_{1} \in \bar{C}$.

Now fix a $c \in C$, and put $e_{c}=e$ the associated idempotent as just constructed and take associated Pierce decomposition, $\mathscr{A}=\mathscr{A}_{1}+\mathscr{A}_{1 / 2}+\mathscr{A}_{0}$, and associated cones $C_{1}$ and $C_{0}$. Let $x \in C$. We know by Lemma 11 that there exists an $r \in \mathscr{R}$ such that $e_{x}=(\exp U(r)) e$, and it is obvious that we may take $r \in \mathscr{A}_{1 / 2}$. Since multiplication by $\exp U(r)$ is an automorphism of $\mathscr{A}$, it is clear that

$$
(\exp U(r)) \mathscr{A}_{1}=\mathscr{A}_{1}\left(e_{x}\right) \text {. }
$$


Write as before $x=x_{1}+x_{0}, x_{1} \in \mathscr{A}_{1}\left(e_{x}\right), x_{0} \in \mathscr{K}$. Then there exists $u \in \mathscr{A}_{1}$ such that $(\exp U(r)) u=x_{1}$. Hence $(\exp U(r))\left(u+x_{0}\right)=x_{1}+x_{0}=x$. Since $\exp U(r)$ is also an automorphism of $C$, it follows that $u+x_{0} \in C$, from which we conclude that $u \in C_{1}$, and $x_{0} \in C_{0}$. Hence we have the following

LEMMA 21. $C$ is contained in the set

$$
S=\bigcup_{r \in \mathscr{A}_{1 / 2}}\left\{\exp U(r) \cdot\left(C_{1}+C_{0}\right)\right\}
$$

Furthermore the closure of $C$ contains $S$.

The next result improves on the last, and gives the exact description of $C$.

THEOREM 22. $C=S$.

Let $x \in C$. Write $x=x_{1}+x_{1 / 2}+x_{0}$, where $x_{1} \in \mathscr{A}_{1}, x_{1 / 2} \in \mathscr{A}_{1 / 2}$ and $x_{0} \in \mathscr{A}_{0}=\mathscr{K}$. There exists $u \in \mathscr{A}_{1}, k \in \mathscr{A}_{0}$, and $r \in \mathscr{A}_{1 / 2}$ such that $(\exp U(r))(u+k)=x$, by the last result, whence $u=x_{1}, r \circ x_{1}=x_{1 / 2}$ and $\frac{1}{2} U^{2}(r) x_{1}+k=x_{0}$. Since $C$ is open, it is possible to select $r \in \mathscr{A}_{1 / 2}$ such that $r \circ x_{1}=x_{1 / 2}^{\prime}$ for all $x_{1 / 2}^{\prime} \in \mathscr{A}_{1 / 2}$ in a suitably small neighborhood of $x_{1 / 2}$, and then since $\mathscr{A}_{1 / 2}$ is a linear space, it is clear that we may select $r \in \mathscr{A}_{1 / 2}$ such that $r \circ x_{1}$ is arbitrary in $\mathscr{A}_{1 / 2}$. With this observation made, it is easy to see that every point of $C_{1}+C_{0}$ is an interior point of $S$. Since $C$ is convex, $C$ is the interior of its closure, and the conclusion of the theorem is immediate.

For any $x \in \mathscr{A}_{1}$, we now define an endomorphism $H(x): \mathscr{A}_{1 / 2} \rightarrow \mathscr{A}_{1 / 2}$ by $H(x) a$ $=2 U(x) a$.

Lemma 23. The map $\mathscr{A}_{1} \rightarrow$ End $\mathscr{A}_{1 / 2}$ given by $x \rightarrow H(x)$ is a representation of the semisimple Jordan algebra $\mathscr{A}_{1}$. If $x \in C_{1}, H(x)$ is nonsingular. $H(e)$ is the identity.

That $H(x)$ is nonsingular for $x \in C_{1}$ is contained in our remarks in the text of the last theorem; i.e., the map $\mathscr{A}_{1 / 2} \rightarrow \mathscr{A}_{1 / 2}$ given by $a_{1 / 2} \rightarrow U\left(x_{1}\right) a_{1 / 2}$ is a surjection. (Let us remark as well that $\mathscr{A}_{1} \cdot \mathscr{A}_{1 / 2} \subset \mathscr{A}_{1 / 2}$ so $\mathscr{A}_{1 / 2}$ is a stable space for $H\left(x_{1}\right)$.)

Now we know from Theorem 6 that $U(x \circ y) r=U(x) U(y) r+U(y) U(x) r$, for any $x, y \in \mathscr{A}$, and $r \in \mathscr{R}$. Taking $x$ and $y$ in $\mathscr{A}_{1}$, and $r$ in $\mathscr{A}_{1 / 2}$, we obtain $H(x \circ y)$ $=(H(x) H(y)+H(y) H(x)) / 2$ which shows that the map gives a representation. Since $U(e) r=\frac{1}{2} r, H(e)$ is the identity.

For $r$ and $s \in \mathscr{A}_{1 / 2}$ define $r \circ s=P(r, s)$.

LeMma 24. (1) $P$ is a bilinear map from $\mathscr{A}_{1 / 2} \times \mathscr{A}_{1 / 2}$ to $\mathscr{K}$.

(2) $P(r, s)=P(s, r)$.

(3) For any $x \in \mathscr{A}_{1}, P(H(x) r, s)=P(r, H(x) s)$.

The first two statements are obvious. For the third start with the fact known from Theorem 6 that $U(r) U(s)=U(s) U(r)$ for $r, s \in \mathscr{R}$. Then for any $x \in \mathscr{A}$, $U(r) U(x) s=U(r) U(s) x=U(s) U(r) x=U(s) U(x) r$, which specializes to (3).

Now we can give a further description of $C$. From our previous results, it follows that $C$ consists of the totality of points $x+U(r) x+\left(U^{2}(r) / 2\right) x+k$ where $x \in C_{1}$, 
$r \in \mathscr{A}_{1 / 2}$, and $k \in C_{0} . C_{0}$ is a regular open convex cone in $\mathscr{A}_{0}(e)$. We introduce in the usual fashion a partial order in $\mathscr{A}_{0}$ induced from the cone $C_{0}$.

In terms of the notations we have already introduced, we may equally well describe $C$ as the totality of points $x+\frac{1}{2} H(x) r+\frac{1}{4} P(r, H(x) r)+k$ with $x, r$, and $k$ as before. Put $H(x) r=2 s . H(x)$ is nonsingular. Hence $C$ is the totality of points $x+s+P\left(H^{-1}(x) s, s\right)+k$ where now $x \in C_{1}, s \in \mathscr{A}_{1 / 2}, k \in C_{0}$. Finally in terms of the partial ordering introduced above, $C$ is precisely the set of points $x+s+k, x \in C_{1}$, $s \in \mathscr{A}_{1 / 2}, k \in \mathscr{A}_{0}$ such that $k>P\left(H^{-1}(x) s, s\right)$.

THEOREM 25. $C$ is the set of points $x+s+k$ in $\mathscr{A}, x \in C_{1}, s \in \mathscr{A}_{1 / 2}, k \in \mathscr{A}_{0}$ such that $k>P\left(H^{-1}(x) s, s\right)$.

In order to complete the description of $C$ we must exploit the geometrical fact that $C$ is convex and does not contain entire straight lines. The result is

THEOREM 26. For any $x \in C_{1}$ and all $s \in \mathscr{A}_{1 / 2}$

(1) $P\left(H^{-1}(x) s, s\right) \in \bar{C}_{0}$

(2) $P\left(H^{-1}(x) s, s\right)=0 \Rightarrow s=0$.

Firstly, if for any $s \neq 0$, and $x \in C_{1}$, we had $P\left(H^{-1}(x) s, s\right)=0$, then $C$ would contain an entire straight line, so second statement follows.

That $C$ be convex is easily seen to require that

$$
P\left(H^{-1}\left(x_{1}\right) s_{1}, s_{1}\right)+P\left(H^{-1}\left(x_{2}\right) s_{2}, s_{2}\right) \geqq P\left(H^{-1}\left(x_{1}+x_{2}\right)\left(s_{1}+s_{2}\right), s_{1}+s_{2}\right)
$$

where $s_{1}$ and $s_{2}$ are arbitrary in $\mathscr{A}_{1 / 2}$ and $x \in C_{1}$. Put $s_{1}=s_{2}=s, x_{1}=\lambda x_{2}=\lambda x, \lambda>0$, to obtain

$$
P\left(H^{-1}(x) s, s\right)+\lambda P\left(H^{-1}(x) s, s\right) \geqq 4 \lambda P\left(H^{-1}(x+\lambda x) s, s\right) .
$$

Let $\lambda \rightarrow 0$ to obtain $P\left(H^{-1}(x) s, s\right) \geqq 0$, which is the first statement.

Note that condition (1) is equivalent to $P(H(x) s, s) \geqq 0$, since we may replace $s$ by $H(x) s$ in (1).

Now we want to establish that our conditions guarantee convexity.

THEOREM 27. Let $V=V_{1} \oplus V_{1 / 2} \oplus V_{0}$ be a direct sum decomposition of vector space $V$. Let $C_{0}$ and $C_{1}$ be regular open convex cones in $V_{0}$ and $V_{1}$ respectively. Let there be given a linear map $x \rightarrow H(x)$ of $V_{1} \rightarrow$ End $V_{1 / 2}$ such that $H(x)$ is nonsingular for $x \in C_{1}$. Let $P$ be a symmetric bilinear map from $V_{1 / 2} \times V_{1 / 2}$ to $V_{0}$ such that $P(H(x) s, s) \in \bar{C}_{0}$ and is zero only for $s=0$. We suppose also that $P(H(x) s, s)$ $=P(s, H(x) s)$. Let $>$ be the partial order in $V_{0}$ induced from the cone $C_{0}$. Then the point set $S$

$$
S=\left\{x+y+z, x \in C_{1}, y \in V_{1 / 2}, z \in V_{0} \mid z>P\left(H^{-1}(x) y, y\right)\right\}
$$

is a regular open convex cone. 
Clearly $S$ is open. For $S$ to be convex is equivalent to the inequality

$$
P\left(H^{-1}\left(x_{1}\right) s_{1}, s_{1}\right)+P\left(H^{-1}\left(x_{2}\right) s_{2}, s_{2}\right) \geqq P\left(H^{-1}\left(x_{1}+x_{2}\right)\left(s_{1}+s_{2}\right), s_{1}+s_{2}\right)
$$

for $x_{1}, x_{2} \in C_{1}, s_{1}, s_{2} \in V_{1 / 2}$.

Replace in the above inequality $s_{1}$ by

$$
H\left(x_{1}+x_{2}\right) H^{-1}\left(x_{2}\right) s_{1}
$$

and $s_{2}$ by $H\left(x_{1}+x_{2}\right) H^{-1}\left(x_{1}\right) s_{2}$. After a little manipulation, the inequality reduces to the equivalent inequality:

$$
\begin{gathered}
P\left(\left\{H^{-1}\left(x_{1}\right)+H^{-1}\left(x_{2}\right)\right\} s_{1}, s_{1}\right)+P\left(\left\{H^{-1}\left(x_{1}\right)+H^{-1}\left(x_{2}\right)\right\} s_{2}, s_{2}\right) \\
\geqq 2 P\left(\left\{H^{-1}\left(x_{1}\right)+H^{-1}\left(x_{2}\right)\right\} s_{1}, s_{2}\right) .
\end{gathered}
$$

Now for any $x \in C_{1}$ and $s_{1}, s_{2} \in V_{1 / 2}$, we have, by hypothesis,

$$
P\left(H^{-1}(x)\left(s_{1}-s_{2}\right), s_{1}-s_{2}\right) \geqq 0,
$$

whence

$$
P\left(H^{-1}(x) s_{1}, s_{1}\right)+P\left(H^{-1}(x) s_{2}, s_{2}\right) \geqq 2 P\left(H^{-1}(x) s_{1}, s_{2}\right),
$$

which establishes truth of above inequality.

We have yet to show regularity of $S$. It is clear that if $x+y+z \in \bar{S}$, with $x \in V_{1}$, $y \in V_{1 / 2}, z \in V_{0}$, that $x \in \bar{C}_{1}, z \in \bar{C}_{0}$. If $S$ is not regular, then there exists a $u \neq 0$ in $V$ such that $u$ and $-u$ both belong to $\bar{S}$. Then we must have $u \in V_{1 / 2}$, since $C_{1}$ and $C_{0}$ are regular. For any point $x+y+z \in S$ we would have then that $x+y+\lambda u+z \in S$, for any real $\lambda$; i.e. $z>P\left(H^{-1}(x)(y+\lambda u),(y+\lambda u)\right)$ which is impossible unless $P\left(H^{-1}(x) u, u\right)=0$, implying $u=0$, and completing the proof.

Let $x_{1}$ and $x_{2} \in V_{1}$. By $H\left(x_{1}+i x_{2}\right)$ we shall understand the endomorphism $H\left(x_{1}\right)+i H\left(x_{2}\right)$ of complexified $V_{1 / 2}$.

LEMMA 28. With the hypothesis of the last theorem, if $x_{1} \in C_{1}$, then $H\left(x_{1}\right)+i H\left(x_{2}\right)$ is nonsingular.

For suppose $\left[H\left(x_{1}\right)+i H\left(x_{2}\right)\right]\left(y_{1}+i y_{2}\right)=0$ for $y_{1}, y_{2} \in V_{1 / 2}$. Then $H\left(x_{1}\right) y_{1}$ $=H\left(x_{2}\right) y_{2}$ and $H\left(x_{1}\right) y_{2}=-H\left(x_{2}\right) y_{1}$; hence

$$
P\left(H\left(x_{1}\right) y_{1}, y_{1}\right)=P\left(H\left(x_{2}\right) y_{2}, y_{1}\right)=P\left(y_{2}, H\left(x_{2}\right) y_{1}\right)=-P\left(H\left(x_{1}\right) y_{2}, y_{2}\right)
$$

and since $C_{0}$ is regular, we conclude that all of above are zero. Hence $y_{1}=y_{2}=0$.

It is convenient now to exhibit the map $w(z)=[I-(i / 2) U(z)]^{-1} z$, which gave rise to this section, making use of the facts and notations already established. The formula given above for $w$ is valid for sufficiently small $z$, where it may be written in form

$$
w(z)=\sum_{v=0}^{\infty} \frac{i^{v}}{2^{v}} z^{v+1}
$$

Let $z=z_{1}+z_{1 / 2}+z_{0}, z_{1} \in \mathscr{A}_{1} \oplus i \mathscr{A}_{1}, z_{2} \in \mathscr{A}_{1 / 2} \oplus i \mathscr{A}_{1 / 2}, z_{0} \in \mathscr{A}_{0} \oplus i \mathscr{A}_{0}$. 
It is easy to establish inductively that for $\nu \geqq 1$ we have

$$
\left(z_{1}+z_{1 / 2}+z_{0}\right)^{\nu}=z_{1}^{\nu}+H^{\nu-1}\left(z_{1}\right) z_{1 / 2}+P\left(H^{\nu-2}\left(z_{1}\right) z_{1 / 2}, z_{1 / 2}\right) .
$$

Hence

$$
\begin{aligned}
w(z)= & {\left[I-(i / 2) U\left(z_{1}\right)\right]^{-1} z_{1}+\left[I-(i / 2) H\left(z_{1}\right)\right]^{-1} z_{1 / 2} } \\
& +(i / 2) P\left(\left[I-(i / 2) H\left(z_{1}\right)\right]^{-1} z_{1 / 2}, z_{1 / 2}\right)+z_{0} .
\end{aligned}
$$

We have already seen, since $\mathscr{A}_{1}$ is semisimple, that $\left[I-(i / 2) U\left(z_{1}\right)\right]^{-1} z_{1}$ extends to a holomorphic map of $C_{1} \oplus i \mathscr{A}_{1}$ onto itself.

Also we have just established that $H\left(z_{1}\right)$ is nonsingular for $z_{1} \in C_{1} \oplus i \mathscr{A}_{1}$. But then $I-(i / 2) H\left(z_{1}\right)=-(i / 2) H\left(z_{1}+2 i e\right)$ is nonsingular. Hence $w$ is defined in $T_{C}$.

We want to show that $w$ maps $T_{C}$ onto itself. Since the inverse map is of the same form, it suffices to show that $w$ maps $T_{C}$ into itself. It is somewhat simpler to consider the map $w^{\prime}(z)=w(z-2 i e)-2 i e$. We find easily

$$
w^{\prime}(z)=4 U^{-1}\left(z_{1}\right) e+2 i H^{-1}\left(z_{1}\right) z_{1 / 2}-P\left(H^{-1}\left(z_{1}\right) z_{1 / 2}, z_{1 / 2}\right)+z_{0}
$$

where by $U^{-1}\left(z_{1}\right) e$ we mean the holomorphic extension of the inverse of $U\left(z_{1}\right)$, restricted to $\mathscr{A}_{1}$, applied to $e$, as described in the text following Theorem 16 .

Let $w^{\prime}(z)=w_{1}+w_{1 / 2}+w_{0}$, and put $z_{1}=x+i y, z_{1 / 2}=r+i s, z_{0}=k+i l$. Then $w_{1}=u+i v=4 U^{-1}\left(z_{1}\right) e, w_{1 / 2}=2 i H^{-1}\left(z_{1}\right) z_{1 / 2}=p+i q$ and $w_{0}=-P\left(H^{-1}\left(z_{1}\right) z_{1 / 2}, z_{1 / 2}\right)$ $+z_{0}=g+i h$.

Given that $k>P\left(H^{-1}(x) r, r\right)$, we want to show that $g>P\left(H^{-1}(u) p, p\right)$. Since $H$ is a representation, we have immediately that $H\left(w_{1}\right)=4 H^{-1}\left(z_{1}\right)$. So $H(x)+i H(y)$ $=\frac{1}{4}[H(u)+i H(v)]^{-1}$, and since $u \in C_{1}, H(u)$ is nonsingular, and we find easily that $H^{-1}(x)=\frac{1}{4}\left[H(u)+H(v) H^{-1}(u) H(v)\right]$. Also $w_{1 / 2}=2 i H^{-1}\left(z_{1}\right) z_{1 / 2}=(i / 2) H\left(w_{1}\right) z_{1 / 2}$, so we find that $p=-\frac{1}{2} H(u) s-\frac{1}{2} H(v) r$, and $q=-\frac{1}{2} H(v) s+\frac{1}{2} H(u) r$. Also

$$
w_{0}=z_{0}-P\left(H^{-1}\left(z_{1}\right) z_{1 / 2}, z_{1 / 2}\right)=z_{0}+(i / 2) P\left(w_{1 / 2}, z_{1 / 2}\right),
$$

so

$$
\begin{aligned}
g & =k-\frac{1}{2} P(p, s)-\frac{1}{2} P(q, r) \\
& =k+\frac{1}{4} P(H(u) s, s)+\frac{1}{4} P(H(v) r, s)+\frac{1}{4} P(H(v) s, r)-\frac{1}{4} P(H(u) r, r) .
\end{aligned}
$$

Now we compute $P\left(H^{-1}(u) p, p\right)$, using the value of $p$ determined above, and find that

$$
P\left(H^{-1}(u) p, p\right)=\frac{1}{4} P(H(u) s, s)+\frac{1}{2} P(H(v) r, s)+\frac{1}{4} P\left(H(v) H^{-1}(u) H(v) r, r\right) .
$$

Hence the inequality $g>P\left(H^{-1}(u) p, p\right)$ simply requires that $k>\frac{1}{4} P(H(u) r, r)$ $+\frac{1}{4} P\left(H(v) H^{-1}(u) H(v) r, r\right)$. But the right-hand side is precisely $P\left(H^{-1}(x) r, r\right)$. Hence we have proved

THEOREM 29. The necessary and sufficient condition that $T_{C}$ have a nonaffine automorphism is that $C$ have the structure of a Jordan cone. If $U(x)$ is the endomorphism giving Jordan multiplication by $x$ then $w(z)=[I-(i / 2) U(z)]^{-1} z$ extends to be an automorphism of $T_{C}$. 
It is possible to carry further the description of Jordan cones, by giving a complete description of the representations of the semisimple algebra $\mathscr{A}_{1}$. We know that $\mathscr{A}_{1}$ is a mutant of a compact Jordan algebra $\mathscr{B}$. If $U(x)$ gives multiplication in $\mathscr{A}_{1}$, and $V(x)$ gives multiplication in $\mathscr{B}$, then we have seen that

$$
U(x)=V(x) V(f)-V(f) V(x)+V(V(x) f)
$$

where $f$ is such that $2 V^{2}(f)-V(V(f) f)$ is a nonsingular endomorphism of $\mathscr{A}_{1}$. This implies that $f$ has an inverse in the compact algebra $\mathscr{B}$. In fact, the inverse of $f$ is the unit for $\mathscr{A}_{1}$.

The algebra $\mathscr{B}$ is known to be the direct sum of simple compact algebras $\mathscr{B}_{i}$, and it is known [2] that every representation of $\mathscr{B}$ is the direct sum of representations of each of the $\mathscr{B}_{i}$. Furthermore, it is known [2] that every representation of $\mathscr{B}$ is a direct sum of irreducible representations.

Finally, since the universal enveloping algebras of compact algebras have been determined [1], [2], it is known that, aside from the trivial representation, a compact simple Jordan algebra, which is not the exceptional algebra, has a unique (up to equivalence) real irreducible representation. The exceptional algebra has, of course, no nontrivial representation.

There is a simple relation between representations of a semisimple algebra, and representations of its semisimple mutants, which we now present. (The mutant is semisimple if the mutating element $f$ has an inverse.)

LEMMA 30. Let $\mathscr{B}$ be a compact algebra, $p$ its unit, and $x \rightarrow L(x)$ a representation of $\mathscr{B}$ such that $L(p)=I$. Then $x \rightarrow H(x)=L(x) L(f)$ is a representation of its mutant $\mathscr{A}_{1}$, such that $H(e)=I . L(f)$ is nonsingular, so the result above sets up a one-one correspondence between (equivalence classes of) representations of $\mathscr{B}$ and representations of $\mathscr{A}_{1}$.

(The condition $L(p)=I$ is essentially superfluous. It is satisfied as soon as we assert that $L$ does not contain the trivial representation.)

For the proof, let us simply compute

$$
L(U(x) y)=L(V(x) V(f) y)-L(V(f) V(x) y)+L(V(y) V(x) f),
$$

which we find to be equal to $(L(x) L(f) L(y)+L(y) L(f) L(x)) / 2$. Hence

$$
\begin{aligned}
H(U(x) y) & =L(U(x) y) L(f) \\
& =(L(x) L(f) L(y) L(f)+L(y) L(f) L(x) L(f)) / 2 \\
& =(H(x) H(y)+H(y) H(x)) / 2 .
\end{aligned}
$$

$f^{-1}$ is a polynomial in $f$, hence $L(f)$ and $L\left(f^{-1}\right)$ commute. Since $L\left(f \circ f^{-1}\right)=L(p)$ $=I$, it follows that $L\left(f^{-1}\right)=L^{-1}(f)$, which completes the proof of the theorem.

(REMARK. Altering a representation to an equivalent one gives a linearly equivalent cone.) 
Now we are faced with the following problem. A representation $H$, without trivial components, of $\mathscr{A}_{1}$ being given, how do we construct the bilinear function $P(\cdot, \cdot)$ satisfying the conditions of Lemma 24 and Theorem 26 . We call such a form a $C_{0}$ positive form for the representation $H$. The problem is ameliorated by the following trivial observation.

LEMma 31. $Q(s, s)$ is a $C_{0}$ positive form for the representation $L(x)$ of the compact Jordan algebra $\mathscr{B}$ if and only if $P(s, s)=Q(s, L(f) s)$ is a $C_{0}$ positive form for the representation $H(x)=L(x) L(f)$ of $\mathscr{A}_{1}$.

The proof is trivial, and is omitted.

Hence, we are reduced to constructing $C_{0}$ positive forms for representations without trivial component of the compact Jordan algebra $\mathscr{B}$. Since every such representation is a direct sum of representations of the simple ideals in $\mathscr{B}$, the more general problem is easily reduced to the problem of constructing $C_{0}$ positive forms for representations without trivial component of a simple nonexceptional Jordan algebra $\mathscr{B}$. It is to this that we now turn our attention.

Let $x \rightarrow K(x)$ be the unique nontrivial real irreducible representation of $\mathscr{B}$, acting in a vector space $V$. Then any representation $L(x)$ of $\mathscr{B}$ is the direct sum of $k$ copies of $K(x)$, so the representation space for $L(x)$ may be identified with $V \otimes W$, where $W$ is any real $k$-dimensional space. $V \otimes W$ is a $\mathscr{B}$ module under the action

$$
x(v \otimes w)=K(x) v \otimes w=(K(x) \otimes I)(v \otimes w) .
$$

From the known structure of the universal enveloping algebra of $\mathscr{B}$, it follows that there is a positive symmetric bilinear form $(\cdot, \cdot)$ on $V$ with respect to which the endomorphisms $K(x)$ are selfadjoint. Let $[\cdot, \cdot]$ be any positive symmetric bilinear form on $W$, and then define a positive symmetric bilinear form $\{\cdot, \cdot\}$ on $V \otimes W$ by setting $\left\{v_{1} \otimes w_{1}, v_{2} \otimes w_{2}\right\}=\left(v_{1}, v_{2}\right)\left[w_{1}, w_{2}\right]$ and extending by linearity.

The endomorphisms $K(x) \otimes I$ are selfadjoint with respect to $\{\cdot, \cdot\}$. Now let $\omega$ be any point in $\mathscr{A}_{0}^{*}$; i.e., $\omega$ is a linear form on the ambient space of $C_{0}$. If $\omega \in C_{0}^{*}$, then $\omega$ is positive on $\bar{C}_{0}-\{0\}$. Consider $\omega(P(r, s)), P$ being a $C_{0}$ positive form for the representation $L(x)=K(x) \otimes I$ of $\mathscr{B}$. We may write $\omega(P(r, s))=\{Q(\omega) r, s\}$. Then $Q(\omega)$ is selfadjoint with respect to $\{\cdot, \cdot\}$ and the map $\omega \rightarrow Q(\omega)$ is obviously linear.

Also, since $P(L(x) r, s)=P(r, L(x) s)$, it follows that $L(x) Q(\omega)$ is selfadjoint with respect to $\{\cdot, \cdot\}$, and further that $L(x) Q(\omega)$ is positive definite when $x \in C_{1}$ and $\omega \in C_{0}^{*}$. Since $L(x) Q(\omega)$ is selfadjoint, we conclude, by taking adjoints, that $L(x) Q(\omega)=Q(\omega) L(x)$.

The commutator algebra of the set of endomorphisms $K(x)$ is isomorphic to the reals, the complexes, or the quaternions, and all three cases arise. Since $K(x)$ is selfadjoint with respect to $(\cdot, \cdot)$, the commutator algebra is stable under the taking of adjoints. From this it follows easily [7] that taking of adjoint in the commutator 
algebra simply corresponds to conjugation (complex or quaternion) for suitable choice of basis.

Suppose for the sake of argument that the commutator algebra is the complex numbers. Let $A$ be an element of the commutator algebra representing $i=\sqrt{ }-1$. Then the adjoint of $A$ is $-A$. Since $(K(x) \otimes I) Q(\omega)=Q(\omega)(K(x) \otimes I)$, it follows immediately that $Q(\omega)$ may be written uniquely in the form $Q(\omega)=I \otimes Q_{0}(\omega)$ $+A \otimes Q_{1}(\omega)$ where $Q_{0}(\omega)$ and $Q_{1}(\omega)$ also depend linearly on $\omega \in \mathscr{A}_{0}^{*} . Q_{0}(\omega)$ is selfadjoint and $Q_{1}(\omega)$ is skew-adjoint with respect to $[\cdot, \cdot]$.

With $Q(\omega)$ as above, then $(K(x) \otimes I) Q(\omega)$ is indeed selfadjoint. We have yet to require that it be positive definite for $x \in C_{1}$ and $\omega \in C_{0}^{*}$. We may write now $L(x) \otimes Q(\omega)=(K(x) \otimes I) Q(\omega)=K(x) \otimes Q_{0}(\omega)+K(x) A \otimes Q_{1}(\omega)$.

Let $r=v_{1} \otimes w_{1}+v_{2} \otimes w_{2}+\cdots+v_{\nu} \otimes w_{v}$. Then

$$
\begin{aligned}
\{(L(x) \otimes Q(\omega)) r, r\}= & \sum_{i}\left(K(x) v_{i}, v_{i}\right)\left[Q_{0}(\omega) w_{i}, w_{i}\right] \\
& +\sum_{i}\left(K(x) A v_{i}, v_{i}\right)\left[Q_{1}(\omega) w_{i}, w_{i}\right] \\
& +2 \sum_{i>j}\left(K(x) v_{i}, v_{j}\right)\left[Q_{0}(\omega) w_{i}, w_{j}\right] \\
& +2 \sum_{i>j}\left(K(x) A v_{i}, v_{j}\right)\left[Q_{1}(\omega) w_{i}, w_{j}\right]
\end{aligned}
$$

shall always be positive for $r \neq 0, x \in C_{1}$, and $\omega \in C_{0}^{*}$. But if $x \in C_{1}$, then it is known that $x$ is the square of an element in $\mathscr{B}$; i.e., $x=y^{2}$. From this it follows, replacing $K(x)$ by $K\left(y^{2}\right)=K^{2}(y)$, that $(K(x) \otimes I) Q(\omega)$ is positive definite for $x \in C_{1}$ if and only if $(I \otimes I) Q(\omega)$ is positive definite.

Since $A$ is skew-adjoint, we have always $(A v, v)=0$. Hence,

$$
\begin{aligned}
\{(I \otimes I) Q(\omega) r, r\}= & \sum_{i}\left(v_{i}, v_{i}\right)\left[Q_{0}(\omega) w_{i}, w_{i}\right] \\
& +2 \sum_{i>j}\left(v_{i}, v_{j}\right)\left[Q_{0}(\omega) w_{i}, w_{j}\right]+2 \sum_{i>j}\left(A v_{i}, v_{j}\right)\left[Q_{1}(\omega) w_{i}, w_{j}\right] .
\end{aligned}
$$

Let $\nu=2$ and $v_{2}=A v_{1}$. Then we must have

$$
\left[Q_{0}(\omega) w_{1}, w_{1}\right]+\left[Q_{0}(\omega) w_{2}, w_{2}\right]-2\left[Q_{1}(\omega) w_{2}, w_{1}\right] \geqq 0
$$

for $\omega \in C_{0}^{*}$, and this is immediately seen to be equivalent to the statement that the endomorphism $Q_{0}(\omega)+i Q_{1}(\omega)$ is Hermitian positive with respect to the Hermitian form extending [ , ] on $W \times W$ to the complexification of the space $W$.

The above necessary condition for positivity is easily seen to be sufficient. Indeed, a basis for $V$ may be chosen to consist of vectors $v_{i}$ and $A v_{i}$ such that

$$
\left(v_{i}, v_{j}\right)=\delta_{i j}, \quad\left(v_{i}, A v_{j}\right)=0, \quad\left(A v_{i}, A v_{j}\right)=\delta_{i j}
$$

and writing any $r \in V \otimes W$ using these basis elements for $V$ gives the general result immediately.

The analogous statements hold for the real and quaternionic cases as well. Since all the steps are reversible, we have 
THEOREM 32. Let the representation of $\mathscr{B}$ be given by $K(x) \otimes I$ in the space $V \otimes W, W$ being of dimension $k$. Let the commutator algebra of $\{K(x)\}$ be $\boldsymbol{P}$, where $\boldsymbol{P}$ is $\boldsymbol{R}, \boldsymbol{C}$, or $\boldsymbol{H}$. Extend the bilinear form [ , ] on $W$ to a sesquilinear form, now denoted [, ]' on $\boldsymbol{P}_{\boldsymbol{R}} \otimes W$. Then the $C_{0}$ positive forms on $V \otimes W$ are in one-one correspondence with the linear maps $\omega \rightarrow Q(\omega)$ from the ambient linear space of $C_{0}^{*}$ into the selfadjoint endomorphisms of $\boldsymbol{P} \otimes W$ with respect to [ , ]' with the property that $Q(\omega)$ is positive for $\omega \in C_{0}^{*}$.

There is a rather interesting way of recasting the last statement, which we now describe. Let $V$ and $W$ be real vector spaces, and let $D$ and $E$ be regular open convex cones in $V$ and $W$ respectively. A map $h \in \operatorname{Hom}(V, W)$ is said to be order preserving if $h D \subset \bar{E}$. The order preserving maps, denoted Ohom $(V, W)$, obviously form a closed convex cone in $\operatorname{Hom}(V, W)$. A map $h$ is called strictly order preserving if $h \in$ Interior Ohom $(V, W)$. Clearly, $h$ is strictly order preserving if and only if $h D \subset E$.

By virtue of the natural identification of $\operatorname{Hom}(V, W)$ with $V^{*} \otimes W$, we may identify $\operatorname{Ohom}(V, W)$ with the subset of $V^{*} \otimes W$ consisting of elements $r=\sum_{i} v_{i}^{*} \otimes w_{i}$ such that $\sum v_{i}^{*}(v) w_{i} \in \bar{E}$ for all $v \in \bar{D}$, which is the same as saying that $\sum v_{i}^{*}(v) w_{i}\left(w^{*}\right) \geqq 0$ for all $v \in \bar{D}, w^{*} \in \bar{E}^{*}$.

In $V \otimes W^{*}$, we may form the open convex cone, denoted $D \otimes E^{*}$, consisting of elements $r=\sum v_{i} \otimes w_{i}^{*}$ such that $v_{i} \in D, w_{i} \in E^{*}$.

It is possible to prove that the closure of $D \otimes E^{*}$ is $\bar{D} \otimes \bar{E}^{*}$, but we do not require this result.

At any rate it is clear that the dual cone to $D \otimes E^{*}$ may be identified with the strictly order preserving maps of $V$ into $W$. Hence

TheOREM 33. Int Ohom $(V, W)=\left(D \otimes E^{*}\right)^{*}$.

The reinterpretation of Theorem 32 in the light of Theorem 33 is obvious. In most instances, however, the description of $D \otimes E^{*}$ is too complicated to shed any light on $\operatorname{Ohom}(V, W)$.

We close this section with an observation which enables us to give a somewhat simpler geometrical description of cones with a Jordan structure. Namely, if $C$ has a Jordan structure given by multiplication $U(x)$, then any mutant of $U(x)$ also equips $C$ with a Jordan structure. Suppose then we let $u$ be a primitive idempotent in $\mathscr{A}_{1}$, and consider the mutant multiplication $V(x)=U(x) U(u)-U(u) U(x)$ $+U(x \circ u)$. The radical of the algebra with this mutant multiplication is now of codimension one, and the semisimple part is of dimension one. Repeating the general analysis for this case, we may arrive at the following conclusion.

THEOREM 34. A regular open convex cone $C$ has Jordan structure if and only if there exists a vector space $V$, a regular open convex cone $D$ in $V$, a vector space $W$ and a symmetric bilinear map $P$ from $W \times W$ to $V$ with the property that $P(w, w) \in \bar{D}$ and is zero only for $w=0$, such that $C$ is linearly equivalent to the set of points $(x, v, w)$ in $\boldsymbol{R}^{+} \times V \times W$ for which $x v-P(w, w) \in D$. 
V. Infinitesimal motions. We can supplement some of the results of [4] on the nature of the Lie algebra of infinitesimal (holomorphic) motions of $T_{C}$.

Denote by $\Theta_{t}$ the automorphism of $T_{C}$ given by $\Theta_{t} z=e^{t} z$. Consider again the automorphism of $T_{C}$ given by $w(z)=[I-(i / 2) U(z)]^{-1} z$ and put

$$
w_{t}(z)=\left(\Theta_{-t} \circ w \circ \Theta_{t}\right)(z)=\left[I-(i / 2) e^{t} U(z)\right]^{-1} z .
$$

On examining the 2-jet of $w_{t}(z)$ at the origin, and using the unicity theorem developed earlier, we immediately obtain

LEMMA 1. $w_{t} \circ w_{s}=w_{t+s}$; i.e., $w_{t}$ is a one parameter subgroup, and the infinitesimal generator is $(i / 2) U(z) z$.

We can give a converse to the last result. Let $W_{t}(z)$ be a one parameter group of automorphisms of $T_{C}$ with infinitesimal generator $q(z) . q(z)=(d / d t) W_{t}(z)(t=0)$. Using the notation already established for the kernel function of $T_{C}$, we know that

$$
L\left(W_{t}\left(z_{1}\right)+\overline{W_{t}\left(z_{2}\right)}\right)\left|\frac{\partial W_{t}\left(z_{1}\right)}{\partial z_{1}}\right|\left|\overline{\frac{\partial W_{t}\left(z_{2}\right)}{\partial z_{2}}}\right|=L\left(z_{1}+\bar{z}_{2}\right) .
$$

On differentiating the last with respect to $t$ and setting $t=0$, we obtain

$$
\left\langle b\left(z_{1}+\bar{z}_{2}\right), q\left(z_{1}\right)+\overline{q\left(z_{2}\right)}\right\rangle+\operatorname{tr}\left(\partial q\left(z_{1}\right) / \partial z_{1}\right)+\overline{\operatorname{tr}\left(\partial q\left(z_{2}\right) / \partial z_{2}\right)}=0,
$$

valid provided $L\left(z_{1}+\bar{z}_{2}\right) \neq 0$.

Differentiate the last with respect to $\bar{z}_{2}$ to obtain

$$
B\left(z_{1}+\bar{z}_{2}\right)\left(q\left(z_{1}\right)+\overline{q\left(z_{2}\right)}\right)+{ }^{t}\left(\overline{\frac{\partial q\left(z_{2}\right)}{\partial z_{2}}}\right) b\left(z_{1}+\bar{z}_{2}\right)+\overline{\frac{\partial}{\partial z_{2}} \operatorname{tr} \frac{\partial q\left(z_{2}\right)}{\partial z_{2}}}=0,
$$

also valid for $L\left(z_{1}+\bar{z}_{2}\right) \neq 0$.

Now for any $z_{1}^{0}$ in $C^{m}$, we can find a $z_{2} \in T_{C}$ such that $z_{1}^{0}+\bar{z}_{2} \in T_{C}, L\left(z_{1}^{0}+\bar{z}_{2}\right) \neq 0$ and $B\left(z_{1}^{0}+\bar{z}_{2}\right)$ is nonsingular. This being so, it is clear that we can solve (I) for $q\left(z_{1}\right)$ for all $z_{1}$ in a sufficiently small neighborhood of $z_{1}^{0}$. Hence we conclude that $q(z)$ is an entire function of $z$.

We can easily conclude much more. Expand $q(z)$ in a power series about $z=0$,

$$
q(z)=A_{0}+A_{1}(z)+\cdots+A_{v}(z)+\cdots
$$

where $A_{v}(z)$ is homogeneous of weight $\nu$. Now in (I) put $z_{2}=0$, and use the fact that $b(z)$ is homogeneous of weight -1 , that $B(z)$ is homogeneous of weight -2 and nonsingular for $z \in C$ to conclude immediately that $A_{v}(z) \equiv 0$ for $v>2$; i.e., $q(z)$ is a polynomial of degree at most 2 .

It is now convenient to write $q(z)$ in the form $q(z)=i a_{0}+A z+(i / 2) U(z) z$ where $U(z)$ is uniquely determined by the requirement that $U\left(z_{1}\right) z_{2}=U\left(z_{2}\right) z_{1}$.

With this notation, equation (I) becomes

$$
\begin{aligned}
B\left(z_{1}+\bar{z}_{2}\right)[i a+ & \left.A z_{1}+(i / 2) U\left(z_{1}\right) z_{1}-i \bar{a}+\bar{A} \bar{z}_{2}-(i / 2) \overline{U\left(z_{2}\right)} \bar{z}_{2}\right] \\
+ & {\left[{ }^{t} \bar{A}-i^{t} \overline{U\left(z_{2}\right)}\right] b\left(z_{1}+\bar{z}_{2}\right)+\bar{\alpha}=0 }
\end{aligned}
$$


where

$$
\alpha=\frac{\partial}{\partial z} \operatorname{tr} \frac{\partial q(z)}{\partial z}=i \frac{\partial}{\partial z} \operatorname{tr} U(z)
$$

is a constant.

Now replace $z_{2}$ by $\lambda \bar{z}_{1}, \lambda$ real, sort out terms according to the powers of $\lambda$, and in each of the resulting terms, sort out according to weight in $z_{1}$, to obtain all of the following:

$$
\begin{aligned}
B(z)(a-\bar{a}) & =0, \\
B(z) A z+{ }^{t} \bar{A} b(z) & =0, \\
(i / 2) B(z) U(z) z+\bar{\alpha} & =0, \\
B(z) \bar{A} z+{ }^{t} \bar{A} b(z) & =0, \\
-i^{t} \overline{U(\bar{z})} b(z)+2 \bar{\alpha} & =0, \\
\left.-(i / 2) B(z) \overline{U(\bar{z})} z-i^{t} \overline{U(\bar{z}}\right) b(z)+\bar{\alpha} & =0 .
\end{aligned}
$$

From (1) we infer $a=\bar{a}$. From (2) and (4) we obtain $A=\bar{A}$. From (5) and (6) we conclude that

$$
(i / 2) B(z) \overline{U(\bar{z})} z+\bar{\alpha}=0,
$$

which compared with (3) gives $\bar{U}(\bar{z})=U(z)$; i.e., $U(z)$ has real coefficients.

Now for every real vector $a$, ia are the contravariant components of an infinitesimal motion; namely the one parameter subgroup $W_{t}(z)=z+t i a$ has $i a$ as its infinitesimal generator.

Also if $w_{t}(z)$ is a one parameter group with infinitesimal generator $q(z)$, then

$$
\bar{w}_{t}(\bar{z}) \text { has infinitesimal generator } \overline{q(\bar{z})} \text {. }
$$

Using this observation and the preceding description of infinitesimal motions, we have

THEOREM 2. The Lie algebra of infinitesimal motions of $T_{C}$ consists of vectors whose coefficients are polynomials of degree at most 2, and is the direct sum of its homogeneous parts of weight 0,1 , and 2. Weight 0 consists of purely imaginary vectors. Weight 1 are linear polynomials with real coefficients, and weight 2 are polynomials with purely imaginary coefficients.

Theorem 2 is a special case of more general statements appearing in [4].

The infinitesimal motions consisting of polynomials of degree at most one are clearly the generators of affine groups of transformations of $T_{C}$. But we can now explain the role of the degree 2 parts. For let $i U(z) z, U(z)=\bar{U}(\bar{z}), U\left(z_{1}\right) z_{2}=U\left(z_{2}\right) z_{1}$ be an infinitesimal motion. To find the associated one parameter subgroup it generates, we must solve the differential system $d w_{t}(z) / d t=i U(w) w$ subject to the initial condition $w_{0}(z)=z$. 
From known theorems on differential equations, we may seek the solution in a neighborhood of $z=0$ in the form of a power series in $t$,

$$
w_{t}(z)=\sum_{v=0}^{\infty} a_{v}(z) t^{\nu}
$$

with $a_{0}(z)=z$. Solving for the coefficients $a_{v}(z)$ recursively readily yields the conclusions that $a_{1}(z)=i U(z) z$ and that for $\nu>1, a_{v}(z)$ is a polynomial with no terms of degree less than 3 . Since the automorphism $w_{t}(z)$ is defined in a neighborhood of $z=0$ and has nonsingular Jacobian there, it is completely determined by its two jet. Hence we conclude that $w_{t}(z)=[I-i t U(z) z]^{-1} z$. So we have

THEOREM 3. If $i U(z) z$ is an infinitesimal motion, $U\left(z_{1}\right) z_{2}=U\left(z_{2}\right) z_{1}$, then the multiplication $x \circ y=U(x) y, x, y \in \boldsymbol{R}^{m}$, gives $C$ the structure of a Jordan cone.

It is clearly of some interest to investigate then the different Jordan structures on a single cone. The answer is quite simple, and we give it now. The result also clarifies the nature of the group of linear automorphisms of $C$.

THEOREM 4. Let $C$ be a regular open convex cone. Then all Jordan structures on $C$ are mutants of a fixed Jordan structure.

Firstly, let $\mathscr{A}$ be a Jordan structure so selected that its radical has minimal dimension. Let $U(x)$ give multiplication in $\mathscr{A}$ and let $\langle R \cdot, \cdot\rangle$ be the trace form for $\mathscr{A}$.

Let $V(x)$ give multiplication by $x$ in a second Jordan structure, and let $\left\langle R^{\prime} \cdot, \cdot\right\rangle$ be the trace form in this structure.

From Theorem 3 of $\S \mathrm{V}$, it follows that $H_{\lambda}(x)=U(x)+\lambda V(x)$ is for any real $\lambda$ multiplication by $x$ in some Jordan structure on $C$. Let $c$ be a fixed point in $C$. Then the radical in the structure associated to $H_{\lambda}(x)$ is precisely the null space of $R_{\lambda}=2 H_{\lambda}^{2}(c)-H_{\lambda}\left(H_{\lambda}(c)\right)$. Now for all sufficiently small $\lambda$, it is clear, by a straightforward compactness argument, that no vector in $\mathscr{A}_{1}$ is annihilated by $R_{\lambda}$. And since $\mathscr{A}$ has minimal radical, it follows that $R_{\lambda}$ annihilates $\mathscr{R}$ for all small $\lambda$. On displaying $R_{\lambda}$ explicitly in terms of $U(c)$ and $V(c)$, it is then clear that $R^{\prime}$ annihilates $\mathscr{R}$. Hence the radical of $V(x)$ contains the radical of $U(x)$, so the range space of $R$ contains the range space of $R^{\prime}$.

Now we know that for any $x \in C$ we have

$$
2 R=B(x)\left\{2 U^{2}(x)-U(U(x) x)\right\}, \quad 2 R^{\prime}=B(x)\left\{2 V^{2}(x)-V(V(x) x)\right\} .
$$

From above, we know there exists an $S$ such that $R^{\prime}=R S$. Hence $2 V^{2}(x)-V(V(x) x)$ $=\left\{2 U^{2}(x)-U(U(x) x)\right\} S$. Now let $e$ be an idempotent for the multiplication $V(x)$ such as described in Lemma 10 of $\S \mathrm{IV}$. Then $\left\{2 V^{2}(x)-V(V(x) x)\right\} e=V(x) x$. Put $S e=f$. Then $V(x) x=\left\{2 U^{2}(x)-U(U(x) x)\right\} f$, and if this identity is polarized, we obtain that $V(x)=U(x) U(f)-U(f) U(x)+U(U(f) x)$; i.e., $V$ is a mutant of $U$. 
The last result makes it possible to give a better description of the group $\mathbb{S S}$ of infinitesimal automorphisms of $C$. To this end let us pick a Jordan structure $\mathscr{A}$ on $C$ with minimal radical, multiplication being given by $U(x)$. Then we know that $i U(z) z$ is an infinitesimal (holomorphic) motion of $T_{C}$. Let $\mathrm{g} \in \mathbb{S}$. Then $\mathrm{g} z$ is also an infinitesimal motion of $T_{C}$. On taking the commutator of the last two, we obtain the infinitesimal motion $2 i U(z) g z-i g U(z) z$, which may be written in the form $i V(z) z$, where $V(z)$ is put equal to $U(z) \mathfrak{g}-\mathfrak{g} U(z)+U(\mathfrak{g} z)$. When so written, we have that $V(z) w=V(w) z$. Hence by Theorem 3 of $\S \mathrm{V}, V(x)$ gives multiplication for a Jordan structure on $C$. By Theorem 4, there exists $f \in \mathscr{A}$ such that $V(x)=U(x) U(f)$ $-U(f) U(x)+U(U(f) x)$. Put $\mathfrak{h}=\mathfrak{g}-U(f) . \mathfrak{h} \in \mathfrak{S}$, and $U(x) \mathfrak{h}-\mathfrak{h} U(x)+U(\mathfrak{h} x)=0$. The last simply says that the map $x \rightarrow \mathfrak{h} x$ is a derivation of $\mathscr{A}$. Hence we have

TheOREM 5. Let $C$ be a cone with Jordan structure $\mathscr{A}$. Let $\mathscr{A}$ have minimal radical, and let $U(x)$ give multiplication in $\mathscr{A}$. Then $\mathbb{B S}$ is spanned by $\{U(a)\}_{a \in \mathscr{A}}$ and some subspace of the derivations of $\mathscr{A}$.

REMARK. Of course commutators $U(a) U(b)-U(b) U(a)$ give derivations which are automorphisms. In the general case there can be other derivations giving infinitesimal automorphisms of $C$.

\section{REFERENCES}

1. G. Birkhoff and P. M. Whitman, Representation of Jordan and Lie algebras, Trans. Amer. Math. Soc. 65 (1949), 116-136. MR 10, 587.

2. F. D. Jacobson and N. Jacobson, Classification and representation of semi-simple Jordan algebras, Trans. Amer. Math. Soc. 65 (1949), 141-169. MR 10, 588.

3. S. Kaneyuki, On the automorphism groups of homeogeneous bounded domains, J. Fac. Sci. Univ. Tokyo Sect. I 14 (1967), 89-130. MR 37 \#3056.

4. W. Kaup, Y. Matsushima and T. Ochiai, On the automorphisms and equivalences of generalized Siegel domains, Amer. J. Math 92 (1970), 475-497.

5. M. Koecher, Jordan algebras and their applications, Lecture Notes, University of Minnesota, Minneapolis, Minn., 1962.

6. O. S. Rothaus, Domains of positivity, Abh. Math. Sem. Univ. Hamburg 24 (1960), 189-235. MR 22 \#12540.

7. - The construction of homogeneous convex cones, Ann. of Math. (2) 83 (1966), 358376. MR 34 \#2029.

8. - Some properties of Laplace transforms of measures, Trans. Amer. Math. Soc. 131 (1968), 163-169. MR 37 \#3050.

9. M. Takeuchi, On infinitesimal affine automorphisms of Siegel domains, Proc. Japan Acad. 45 (1969), 590-594.

10. È. B. Vinberg, S. G. Gindikin and I. I. Pjateckiī-Sapiro, Classification and canonical realization of complex bounded homogeneous domains, Trudy Moskov. Mat. Obšč. 12 (1963), 359-388 = Trans. Moscow Math. Soc. 1963, 404-437. MR 28 \#1638.

Cornell University, Ithaca, New York 14850 